\title{
Origins of Clustering of Metalate-Extractant Complexes in Liquid-Liquid Extraction
}

Srikanth Nayak, ${ }^{1}$ Raju R. Kumal, ${ }^{1}$ Zhu Liu, ${ }^{2}$ Baofu Qiao, ${ }^{1}$ Aurora E. Clark, ${ }^{2}$ and Ahmet Uysal ${ }^{1,}$ *

${ }^{1}$ Chemical Sciences and Engineering Division, Argonne National Laboratory, Lemont, IL 60439

${ }^{2}$ Department of Chemistry, Washington State University, Pullman, WA 99164

KEYWORDS

Liquid-liquid extraction, small angle X-ray scattering, complex fluids, amphiphile aggregation, vibrational sum frequency generation, ion hydration 
Effective and energy efficient separation of precious and rare metals is very important for a variety of advanced technologies. Liquid-liquid extraction (LLE) is a relatively less energy intensive separation technique, widely used in separation of lanthanides, actinides, and platinum group metals (PGMs). In LLE, the distribution of an ion between an aqueous phase and an organic phase is determined by enthalpic (coordination interactions) and entropic (fluid reorganization) contributions. The molecular scale details of these contributions are not well understood. Preferential extraction of an ion from the aqueous phase is usually correlated with the resulting fluid organization in the organic phase, as the longer-range organization increases with metal loading. However, it is difficult to determine the extent to which organic phase fluid organization causes, or is caused by, metal loading. In this study, we demonstrate that two systems with the same metal loading may impart very different organic phase organization; and investigate the underlying molecular scale mechanism. Small angle X-ray scattering shows that the structure of a quaternary ammonium extractant solution in toluene is affected differently by the extraction of two metalates (octahedral $\mathrm{PtCl}_{6}{ }^{2-}$ and square-planar $\mathrm{PdCl}_{4}{ }^{2-}$ ), although both are completely transferred into the organic phase. The aggregates formed by the metalate-extractant complexes (approximated as reverse micelles) exhibit more long-range order (clustering) with $\mathrm{PtCl}_{6}{ }^{2-}$ compared to that with $\mathrm{PdCl}_{4}{ }^{2-}$. Vibrational sum frequency generation spectroscopy, and complimentary atomistic molecular dynamics simulations on model Langmuir monolayers, indicate that the two metalates affect the interfacial hydration structures differently. Further, the interfacial hydration is correlated with water extraction into the organic phase. These results support a strong relationship between the organic phase organizational structure and different local hydration present within the aggregates of metalateextractant complexes, which is independent of metalate concentration. 


\section{INTRODUCTION}

Chemical separations are fundamental processes involved in transforming raw materials into finished products. ${ }^{1-3}$ Development of cheaper and greener separation processes is a necessity for sustainable industrial technologies. Liquid-liquid extraction (LLE) is a widely used separation process that is relatively less energy-intensive compared to other separation processes such as distillation which is the dominant chemical separation process in industry. ${ }^{2-3}$ Although novel separation methods are being developed, ${ }^{4-9}$ LLE is the dominant separation technology in industrial settings for purification of metals from their ores. With an increasing demand for materials recycling and other important separation tasks, ${ }^{10-11}$ LLE is gaining increased attention. ${ }^{3}$ The principle behind LLE is the preferential partitioning of a solute between two immiscible phases and thus it involves interfacial transport of the solute. In LLE an aqueous solution (the "aqueous phase") of multiple metals is contacted with an organic solution (the "organic phase"). Typically, the organic phase contains surface active extractant molecules that enhance the partitioning and separation of desired metals through a direct, and preferential, interaction with the metal of interest. The aqueous phase speciation of the metal ions, the strength and the specificity of interaction between the extractants and the solutes, and the organization of extractant-metal complexes in the organic phase are expected to affect the distribution and selectivity of the LLE process. However, the molecular scale details of these interactions are not well understood.

The extractants are typically amphiphilic and populate the interface between the immiscible phases in LLE. There is a macroscale interface between the aqueous and the organic phases that the solutes must cross during LLE, which contains important molecular scale organizational features. ${ }^{12-18}$ In addition to this macroscale interface, it is hypothesized that there are nanoscale interfaces within the organic phase in the form of reverse micellar aggregates. ${ }^{19-20}$ The pertinence of this colloidal model of the organic phase is still debated ${ }^{21-22}$ and is possibly system dependent. Regardless of the existence of well-defined nanoscale interfaces in the organic phase, there is strong evidence showing that the extractant aggregation affects the efficiency of LLE. ${ }^{20,23-26}$ Changes to aggregation structure that include increasing correlations and elongation of aggregates have been associated with increased metal and acid extraction. ${ }^{19,}{ }^{23,}{ }^{27-31}$ In addition, the coordination environment around the metal species in the organic phase has been linked to changes in the mesoscale structure of the organic phase. ${ }^{32}$ Although the direction of causality is not known, an interplay between extraction and aggregation has been reported. ${ }^{24}$ There, the extracted metal seeds the formation of reverse micellar aggregates, which in turn enhances the metal extraction has been reported. ${ }^{24}$

Selectivity in LLE is hypothesized to be, in part, driven by the differences in the outer-sphere interactions ${ }^{33}$ and the structure of the organic phases formed after extraction. ${ }^{20,34}$ The weak interactions between the aggregates, which may vary between similar metal-extractant complexes, can affect separation. ${ }^{35}$ These interactions are typically ascribed to van der Waal forces between the polar cores of the aggregates, but more specific intermolecular interactions have also been suggested. ${ }^{36-37}$ These interactions can vary with the nature (identity) of the metal in the organic phase. Thus, while highly selective extractants are being developed for LLE from a coordination chemistry perspective, ${ }^{38-41}$ the effects of long-range interactions need to be considered. 
(a) Nanoscale structure of organic phase (SAXS)

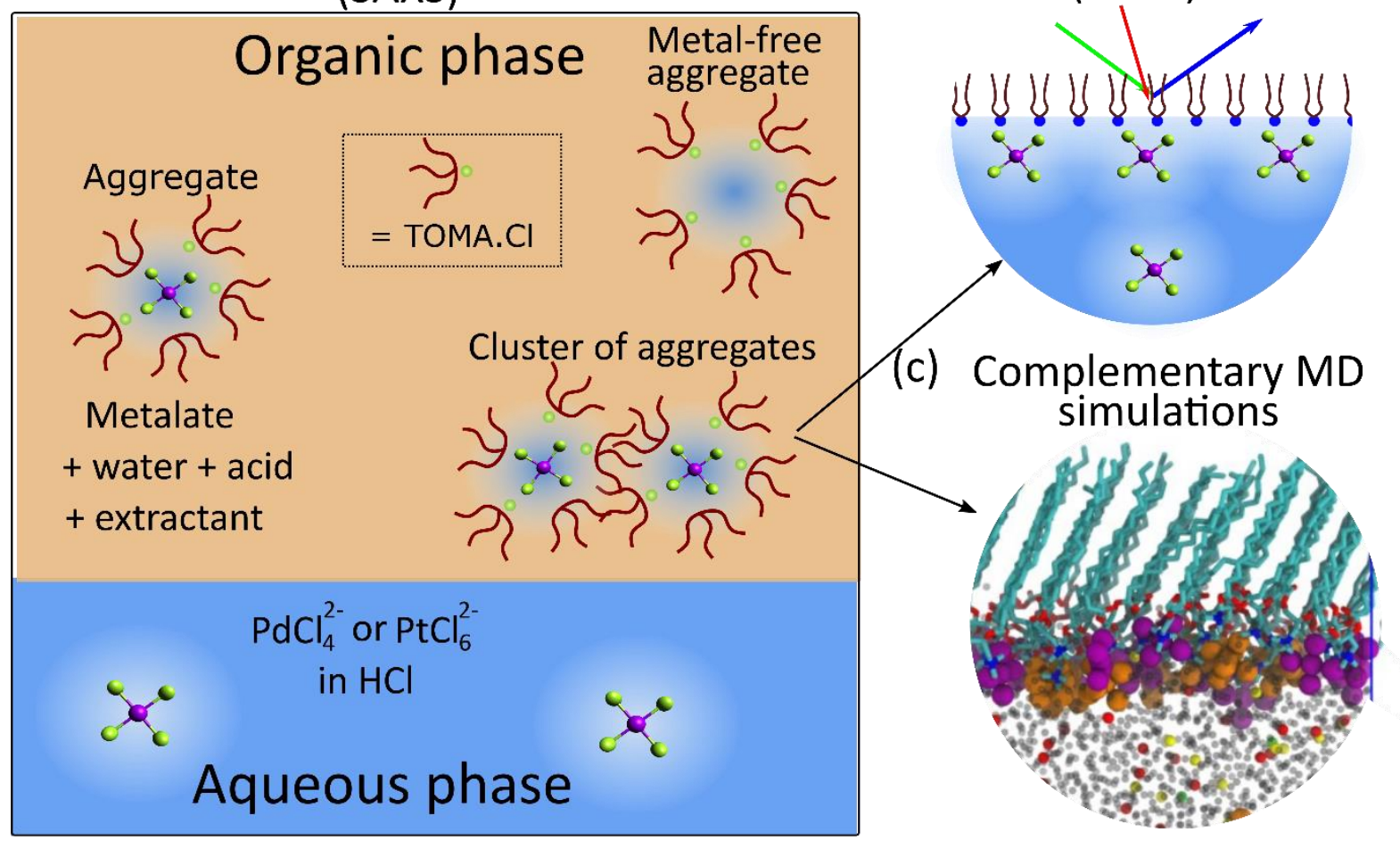

Figure 1. Liquid-liquid extraction (LLE) of a solute $\left(\mathrm{PdCl}_{4}{ }^{2-}\right.$ or $\left.\mathrm{PtCl}_{6}{ }^{2-}\right)$ affects the structure of the organic phase containing the extractant molecules (represented in brown squiggly lines). The extracted species and the extractant molecules form "aggregates" which, in turn, may interact with each other to form "clusters". We examine the role of the interfacial structure in LLE using the techniques depicted above (a) extractant-ion aggregation is studied with small angle X-ray scattering (SAXS) and analyzed with a clustering model; (b) solute effects on interfacial hydration are studied using vibrational sum frequency generation (VSFG) spectroscopy and (c) complementary molecular dynamics simulations of model Langmuir monolayers.

In summary, the organic phase structure can vary with the metallic species, concentration of metal, water and acid. Most studies have focused on the effect of metal content in the organic phase. Here, we study the differences in organizational structure of organic phases having similar metal loading, but different metallic species, namely $\mathrm{PdCl}_{4}{ }^{2-}$ and $\mathrm{PtCl}_{6}{ }^{2-}$. Aliquat 336 is a mixture of quaternary ammonium extractants and is used in the LLE of platinum group metals from their chloride solutions. ${ }^{42-44}$ Quaternary ammonium based extractants are common components of ionic liquids and are frequently diluted with organic

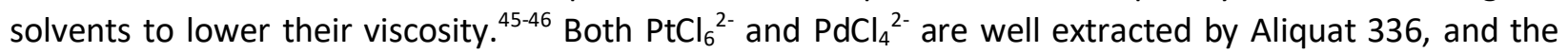
extraction depends on the medium conditions such as background salt concentration. ${ }^{44,47-49}$ Quaternary ammonium extractants are highly aggregated in the organic phase and this aggregation is correlated with their LLE behavior. ${ }^{44,50}$ These extractants typically extract metals by outer-sphere interactions with anionic complexes of the metals. ${ }^{51-52}$ Hydration behaviors of these metalates have been linked with their LLE using basic extractants like Aliquat 336. ${ }^{53-54}$ However, the relation between hydration of the metalates, their extraction, and organic phase aggregation remain elusive as it is difficult to conduct controlled 
experiments; most of these parameters are interrelated and difficult to control individually while keeping others constant. The chlorometalate system presented here, provides a great opportunity to study the different organic phase structuring under equal amount of metal loading.

Extraction of metalates with Aliquat 336 proceeds with co-extraction of water and acid. ${ }^{53,55-57}$ In accordance with the literature, ${ }^{31,50,58-59}$ we define the divalent chloro complexes of platinum and palladium as metalates. The species formed by metalate and two extractant molecules (required for charge balance) are termed metalate-extractant complexes. We define the structures formed by all the extracted species and the extractant molecules as aggregates; and the larger-scale structures formed by multiple interacting aggregates as clusters (Figure 1a). We use trioctylmethylammonium chloride (TOMA.Cl, the main constituent of Aliquat 336) as the extractant. The electrostatic interaction between positively charged quaternary ammonium molecules and the negatively charged metalates is the dominant interaction expected to affect the structure of the organic phase. It is difficult to isolate the effect of this interaction between extractants and the metalates in the organic phase. Therefore, separately, we study the effect of metalates on a positively charged monolayer as a model system. We use 1,2-dipalmitoyl-3-trimethylammonium-propane (DPTAP) as the analogue of TOMA.Cl for the interfacial studies. Organic phase structure has been monitored by small angle X-ray scattering (SAXS) (Figure 1a) and the interfacial interaction between the extractant and the metalates has been studied by X-ray fluorescence near total reflection (XFNTR), ${ }^{60}$ vibrational sum frequency generation (VSFG) spectroscopy (Figure 1b), and atomistic molecular dynamics (MD) simulations, using a model system at the vapor/surfactant/electrolyte interface (Figure 1c). Although both metalates are extracted equally, the organic phase structures with the two metalates are different. The model fits to SAXS data suggests that the difference originates from the different clustering behaviors of aggregates with $\mathrm{PdCl}_{4}{ }^{2-}$ and $\mathrm{PtCl}_{6}{ }^{2-}$ (See Figure 1a for the definition of aggregate and cluster). XFNTR data and MD simulations show that the enthalpic interaction between the extractants and the metalates are almost identical for $\mathrm{PdCl}_{4}{ }^{2-}$ and $\mathrm{PtCl}_{6}{ }^{2-}$, and possibly the main driver of the extraction in this system. VSFG experiments and MD simulations show that the interfacial hydration is significantly different, a feature that could be carried into the organic phase, leading to the different aggregation trends observed in SAXS data. 


\section{RESULTS AND DISCUSSIONS}

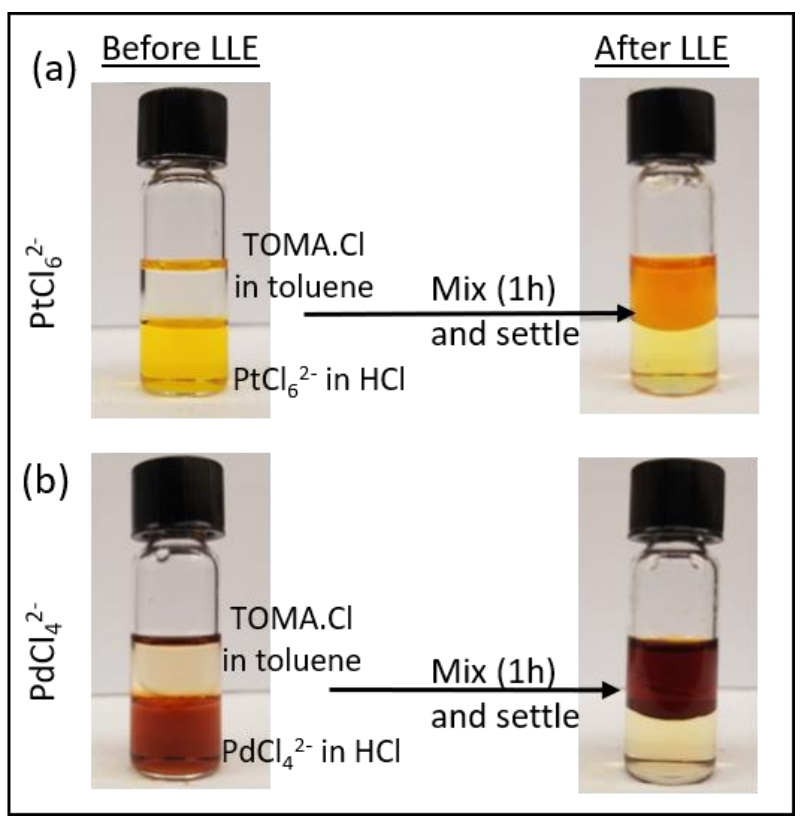

Figure 2. Liquid-liquid extraction (LLE) of (a) $\mathrm{PtCl}_{6}{ }^{2-}$ and (b) $\mathrm{PdCl}_{4}{ }^{2-}$ from $5 \mathrm{M} \mathrm{HCl}$ medium using $0.2 \mathrm{M}$ of TOMA.Cl in toluene. Aqueous phase concentrations of the metalates before and after LLE are tabulated in Table S1.

Extractant Organization in Organic Phase. We conducted LLE of varying concentrations of $\mathrm{PtCl}_{6}{ }^{2-}$ and $\mathrm{PdCl}_{4}{ }^{2-}$ in $5 \mathrm{M} \mathrm{HCl}$ media using $0.2 \mathrm{M} \mathrm{TOMA.Cl}$ (Figure 2). The organic phase was collected after extraction and studied with SAXS, to investigate the differences in nanoscale structure of the organic phases containing $\mathrm{PdCl}_{4}{ }^{2-}$ and $\mathrm{PtCl}_{6}{ }^{2-}$. Composition and speciation of various components in the organic phase after LLE are challenging to quantify. ${ }^{3,61}$ We have measured total metal content and water content to aid understanding of the nanostructure of the organic phase. Inductively coupled plasma mass spectrometry (ICP-MS) analysis of the aqueous phase before and after LLE showed near complete extraction of both $\mathrm{PtCl}_{6}{ }^{2-}$ and $\mathrm{PdCl}_{4}{ }^{2-}$, even at $0.1 \mathrm{M}$ initial aqueous phase concentration (Table S1). Figure 3a and $\mathbf{3 b}$ show the SAXS (in absolute units) from the solutions obtained with LLE of different concentrations of $\mathrm{PdCl}_{4}{ }^{2-}$ and $\mathrm{PtCl}_{6}{ }^{2-}$, respectively. As expected, both the metalates are well extracted with TOMA. ${ }^{62}$ 

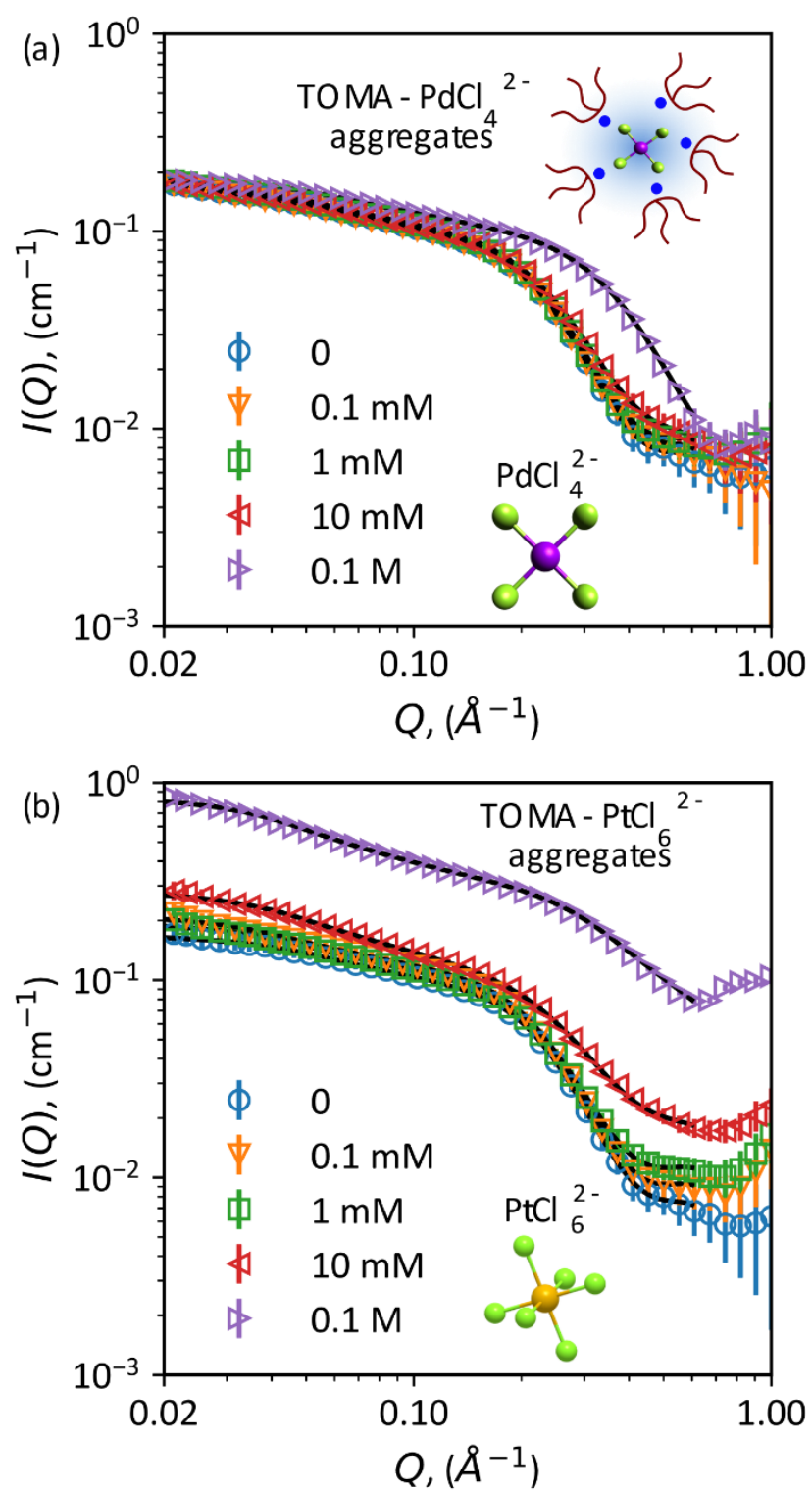

Figure 3. SAXS profiles of organic phases obtained by LLE of (a) $\mathrm{PdCl}_{4}{ }^{2-}$ and (b) $\mathrm{PtCl}_{6}{ }_{6}^{2-}$ from $5 \mathrm{M} \mathrm{HCl}$ medium with $0.2 \mathrm{M}$ of TOMA.Cl in toluene. Legend entries refer to the aqueous phase concentrations of the metalate before LLE. Increasing metal concentration in the organic phase differently affects the organic phase structure in the case of $\mathrm{PdCl}_{4}{ }^{2-}$ and $\mathrm{PtCl}_{6}{ }^{2-}$.

Solutions of TOMA.Cl in toluene that are not contacted with any aqueous phase show aggregation. ${ }^{59}$ SAXS of these solutions show that the aggregates behave like hard-spheres with repulsion-dominant interactions between the spheres. ${ }^{59}$ The organic phase obtained after LLE of metalate-free aqueous phase shows significant structuring due to aggregation of extractants (Figure 3a, blue circles). In comparison to the uncontacted TOMA.Cl solutions, the organic phase obtained after contact with metalate-free aqueous phase shows increased scattering in low- $Q$ region. This increased low- $Q$ scattering is an indication of increasing attractive interactions between the aggregates. ${ }^{27,59}$ 
The differences between the uncontacted and the contacted metalate-free organic phases are likely due to the differences in water and acid content in the organic phases. ${ }^{63}$ When metalates are extracted, there is no appreciable change in the SAXS pattern with $\mathrm{PdCl}_{4}{ }^{2-}$ until the aqueous phase concentration is above $0.01 \mathrm{M}$ (Figure 3a). For charge neutralization, we expect two TOMA ${ }^{+}$to interact with one $\mathrm{PdCl}_{4}{ }^{2-}$, but the aggregation number can be larger as TOMA.Cl pairs can also be present in the aggregate (Figure 1a). Assuming that the aggregation number remains the same with and without the metal, most of the aggregates are metal free at $0.01 \mathrm{M}\left[\mathrm{PdCl}_{4}{ }^{2-}\right]_{\text {aq }}$. This could be the reason behind such relative invariance of the SAXS profiles. At $0.1 \mathrm{M}$ of $\left[\mathrm{PdCl}_{4}{ }^{2-}\right]_{\text {aq }}$ the minimum in the SAXS profile shifts to higher $\mathrm{Q}$ values. With $\mathrm{PtCl}_{6}{ }^{2-}$ a qualitatively different trend appears with increasing $\left[\mathrm{PtCl}_{6}{ }^{2-}\right]_{\text {aq }}$ (Figure 3b). There is an abrupt change above $\left[\mathrm{PtCl}_{6}{ }^{2-}\right]_{\text {aq }}=0.01 \mathrm{M}$, a similarity with $\mathrm{PdCl}_{4}{ }^{2-}$, but there is an increase in overall scattering unlike the $\mathrm{PdCl}_{4}{ }^{2-}$ case.

This abrupt change in SAXS profiles above $0.01 \mathrm{M}$ metalate concentration correlates with the two-step adsorption of these chlorometalates at positively charged interfaces, where interfacial adsorption of chlorometalates qualitatively change around the same relative concentration. ${ }^{12,14}$ This suggests that the trends observed at the nanoscale interfaces in the organic phase could be related to ion-extractant interactions at the macroscopic interfaces. ${ }^{18,64-66}$
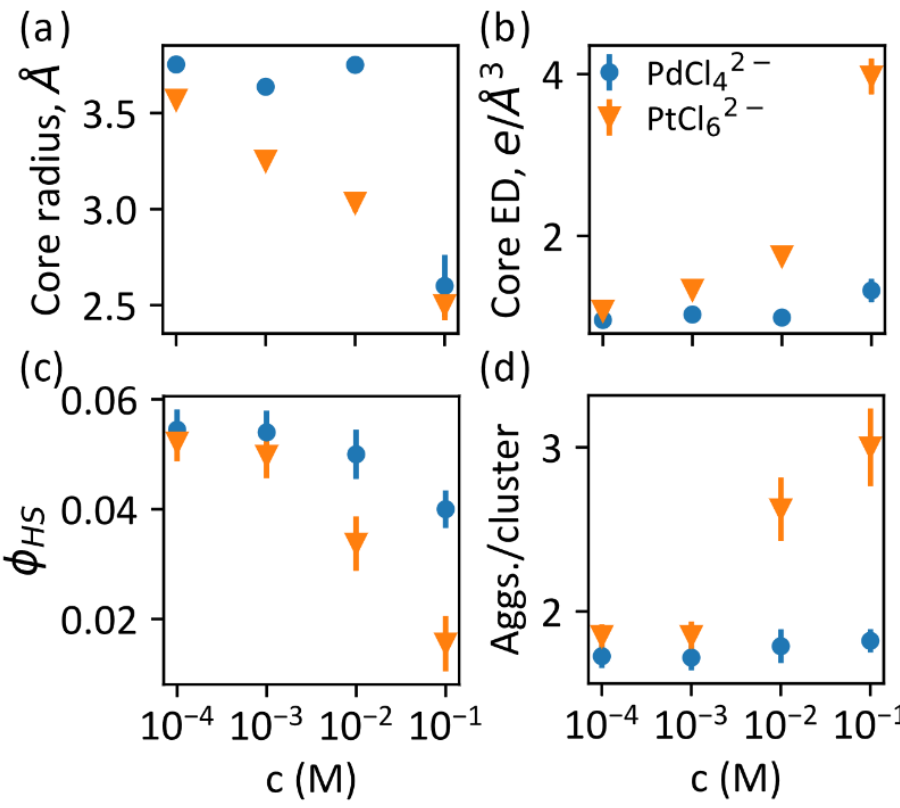

(d)

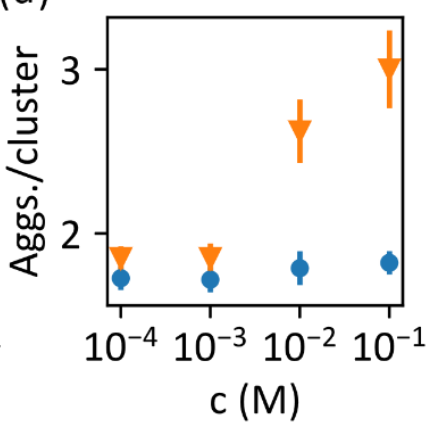

Figure 4. Variation of selected parameters from clustering model fits to SAXS data shown in Figure 3. Blue filled circles and orange triangles correspond to $\mathrm{PdCl}_{4}{ }^{2-}$ and $\mathrm{PtCl}_{6}{ }^{2-}$, respectively. The variation of (a) core radius of the aggregate, (b) electron density of the core, (c) the apparent hard-sphere volume fraction, and (d) aggregates per cluster are shown. The major difference between $\mathrm{PdCl}_{4}{ }^{2-}$ and $\mathrm{PtCl}_{6}{ }^{2-}$ appears in the variation of number of aggregates per cluster.

Clustering of metalate-extractant aggregates. The qualitative differences in SAXS profiles refer to different effects of $\mathrm{PdCl}_{4}{ }^{2-}$ and $\mathrm{PtCl}_{6}{ }^{2-}$ on the nanoscale structure of the organic phase obtained after LLE. (Figure 3). We use a clustering model to understand the origin of these differences. Owing to the amphiphilic nature of the extractants, reverse micellar aggregates (due to extractant-extractant 
interactions) are observed in the organic phase. Since LLE typically involves a high extractant concentration in the organic phase ( $>10 \%$ by volume), the interactions between these aggregates can significantly affect the extraction and the organic phase structure. ${ }^{36}$ This nanoscale structure has been commonly analyzed with the Baxter model of interacting spherical aggregates. Recently, there have been reports describing the inadequacy of this model. ${ }^{21,67}$ Thus, we have adapted a clustering model to describe the organic phase structure. ${ }^{68}$ We have recently showed that this model is very successful in describing how TOMA-lanthanide aggregates cluster under similar conditions. ${ }^{59}$ There are two main advantages of this model compared to similar attempts in the literature. First, it can adequately fit the data represented in absolute units, without the need for an arbitrary scaling factor, providing physically meaningful parameters. Second, it is a simple analytical expression which does not require any computational work to describe the fine details of the aggregates.

In the clustering model, a shape function that defines the finite shape of a cluster of aggregates held together by an attractive interaction is superimposed on the theoretical structure predicted in the absence of such attractive interactions. So, the effective structure of the complex fluid is a result of the repulsive hard-sphere interactions and the attractive interactions between them.

In our SAXS model (see Methods) we assume that all the extractant molecules are aggregating into monodisperse structures and contributing to SAXS. This is a simplifying assumption as the amphiphile aggregation phenomena in organic solvents is not as well defined as in aqueous media. ${ }^{69}$ Further, we have used an average structure factor that describes the interactions between aggregates, whether they contain the metal or not. We also explored other commonly used models (Baxter's sticky hard sphere model, and repulsive ellipsoidal particles), which were comparatively inadequate (Figure S1). Further, we ruled out the possibility that the abrupt change in SAXS data as a function of metal loading is only a result of increasing electron density at the core of the aggregates. The variations in the structures of the organic phases could not be explained solely by variations in the form factor of the aggregates (Figure S2), indicating that the interaction between the aggregates is a function of organic phase composition. Thus, the clustering model provides an analytical method to capture the key trends in the SAXS data. The employment of the clustering model is validated by the black solid lines in Figure 3, which fit well with the experimental SAXS data. The clustering model describes the structure of the organic phase well in all the samples studied here, for most of the Q-range studied $\left(Q<0.6 \AA^{-1}\right)$.

The fit parameters are listed in Table S2. Variation of four of these parameters with aqueous phase metalate $\left(\mathrm{PdCl}_{4}{ }^{2-}\right.$ or $\left.\mathrm{PtCl}_{6}{ }^{2-}\right)$ concentration is shown in Figure 4. Core radius of the aggregates decreases with increasing aqueous phase metal concentration for both $\mathrm{PdCl}_{4}{ }^{2-}$ and $\mathrm{PtCl}_{6}{ }^{2-}$, while the core electron density increases. Although the increase in electron density of the core is commensurate with increasing concentration of metal in the organic phase, the decrease in core radius is interesting. Apparently, metalate-free aggregates do not simply incorporate metalates in their pre-existing structures but lose water and chloride leading to a decrease in the core radius. Karl Fischer analysis of the organic phases (discussed in next subsection), shows that there, indeed, is a decrease in water content with metalate extraction, for both $\mathrm{PtCl}_{6}{ }^{2-}$ and $\mathrm{PdCl}_{4}{ }^{2-}$. A decrease in extracted water with increasing metal extraction has been observed with TOMA in previous studies. ${ }^{70}$ This in contrast with neutral extractants, in which the extracted metal amount is positively correlated with the extracted water. ${ }^{33}$ 
The apparent hard-sphere volume fraction decreases with metal concentration, indicating the predominance of attractive interactions at higher metal concentrations. The clustering of the aggregates increases with the metalate concentration in the case of $\mathrm{PtCl}_{6}{ }^{2-}$; whereas it remains almost constant with varying $\left[\mathrm{PdCl}_{4}{ }^{2-}\right]_{\mathrm{aq}}$. Thus, increasing metal concentration in the organic phase increases the scattering contrast as expected, and also changes the aggregation behavior in the organic phase. In summary, while $\mathrm{PdCl}_{4}{ }^{2-}$ affects the shape of the aggregate alone (reducing core radius), $\mathrm{PtCl}_{6}{ }^{2-}$ affects both the long-range structure (clustering) and the aggregate shape. $\mathrm{PtCl}_{6}{ }^{2-}$ leads to higher clustering than $\mathrm{PdCl}_{4}{ }^{2-}$, especially at the highest metalate loading.

It is important to note that our model does not distinguish between multiple aggregates coming together and forming a cluster or having a single elongated aggregate. In fact, ellipsoidal hardspheres model fits the high- $\mathrm{PtCl}_{6}{ }^{2-}$ data well (Figure S2), although it is not adequate to describe the low-metalate (low clustering) data. The SAXS data clearly shows that individual aggregate structures somewhat deform as they form clusters because there are no peaks corresponding to long-range ordering. A nondeformable, hard-sphere interaction would lead to the appearance of clear peak corresponding to hard-sphere size. ${ }^{59}$ In our SAXS data, with increasing metalate content, there is no enhancement of a hard-sphere peak, suggesting that the aggregates are deformable.

Water Extraction. Metal extraction is frequently correlated, positively or negatively, with water extraction. In quaternary ammonium and tertiary amine extraction systems, water extraction is inversely correlated with metal extraction. We studied the water content in metalate free organic phase $(5 \mathrm{M} \mathrm{HCl}$ contacted) and highest metalate loaded organic phases (Table S1). A significant amount of water ( $1 \mathrm{M}$ ) in metalate free, acid-contacted organic phase. With metalate extraction, the water content is reduced. Interestingly, the $\mathrm{PtCl}_{6}{ }^{2-}$ loaded organic phase is almost four times poorer in water concentration (at 0.04 M) compared to the $\mathrm{PdCl}_{4}{ }^{2-}$ loaded organic phase (at $0.18 \mathrm{M}$ ). While there are $\sim 2$ water molecules per extracted $\mathrm{PdCl}_{4}{ }^{2-}$, there is less than one water per extracted $\mathrm{PtCl}_{6}{ }^{2-}$.

At low metalate concentrations in the aqueous phase the organic phase structures are similar (Figure 3) with both the metalates, but the organic phase metalate concentrations are slightly different (Table S1). Although the minor differences in organic phase concentrations (and hence distribution ratios) could cause difference in the organic phase structure, we expect the difference in water content to be a major factor. The difference in water content between the metalate-loaded organic phases suggests that the interaction between the metalates and water, in the vicinity of the extractant, are different for $\mathrm{PtCl}_{6}{ }^{2-}$ and $\mathrm{PdCl}_{4}{ }^{2-}$. We discuss our interfacial experiments below to probe this further.

Interfacial Studies. The main structural difference between $\mathrm{PdCl}_{4}{ }^{2-}$ and $\mathrm{PtCl}_{6}{ }^{2-}$ is that while the former is a square-planar complex, the latter is an octahedral complex. Consequently, there are differences in hydration structures of these complexes in water. ${ }^{71}$ What are the implications of these differences on the structure of the organic phases containing them? In the SAXS results shown above there appears to be a difference between $\mathrm{PdCl}_{4}{ }^{2-}$ and $\mathrm{PtCl}_{6}{ }^{2-}$ samples at $\mathrm{Q}>0.6 \AA^{-1}$. It is difficult to reliably model this region due to the low signal of scattering contributions from solvent structure and the extractant tail-group structures. To study the interaction between the ions and the extractant head-group, we have used a model system - adsorption of $\mathrm{PdCl}_{4}{ }^{2-}$ and $\mathrm{PtCl}_{6}{ }^{2-}$ to a monolayer of 1,2-dipalmitoyl-3trimethylammonium-propane (DPTAP) at the air/aqueous interface (Figure 1b). Langmuir monolayers are often used as model systems to study the behavior of biological membranes ${ }^{72-73}$ and fluid interfaces found 
in LLE. ${ }^{13-14,66,74}$ DPTAP forms a positively charged Langmuir monolayer on aqueous subphases mimicking the interfaces populated by TOMA ${ }^{+}$in the reverse micellar aggregates. All the samples in the model system contain $0.5 \mathrm{M} \mathrm{LiCl}$ maintained at $\mathrm{pH}=2$ with $\mathrm{HCl}$. We verified that DPTAP is indeed a good model monolayer to study the effect of the chlorometalates by XFNTR and MD studies. Both studies showed that the number density of $\mathrm{PdCl}_{4}{ }^{2-}$ and $\mathrm{PtCl}_{6}{ }^{2-}$ are equal at the interface (Figure $\mathrm{S} 3$ ), which indicates similar enthalpic interaction strength between the quaternary amine headgroups and both metalates.

The equal adsorption of $\mathrm{PtCl}_{6}{ }^{2-}$ and $\mathrm{PdCl}_{4}{ }^{2-}$ at DPTAP is of note as these two complexes differ in their hydration structures, which typically affects interfacial adsorption behaviors. ${ }^{75}$ Since XFNTR only reports on the interfacial coverage of the metalate ions, we conducted VSFG spectroscopy of the model DPTAP system to study the interfacial hydration structure. VSFG is a surface-specific, second order non-linear spectroscopic technique that probes the orientational order of the dipoles near the interface. ${ }^{76-80}$ 

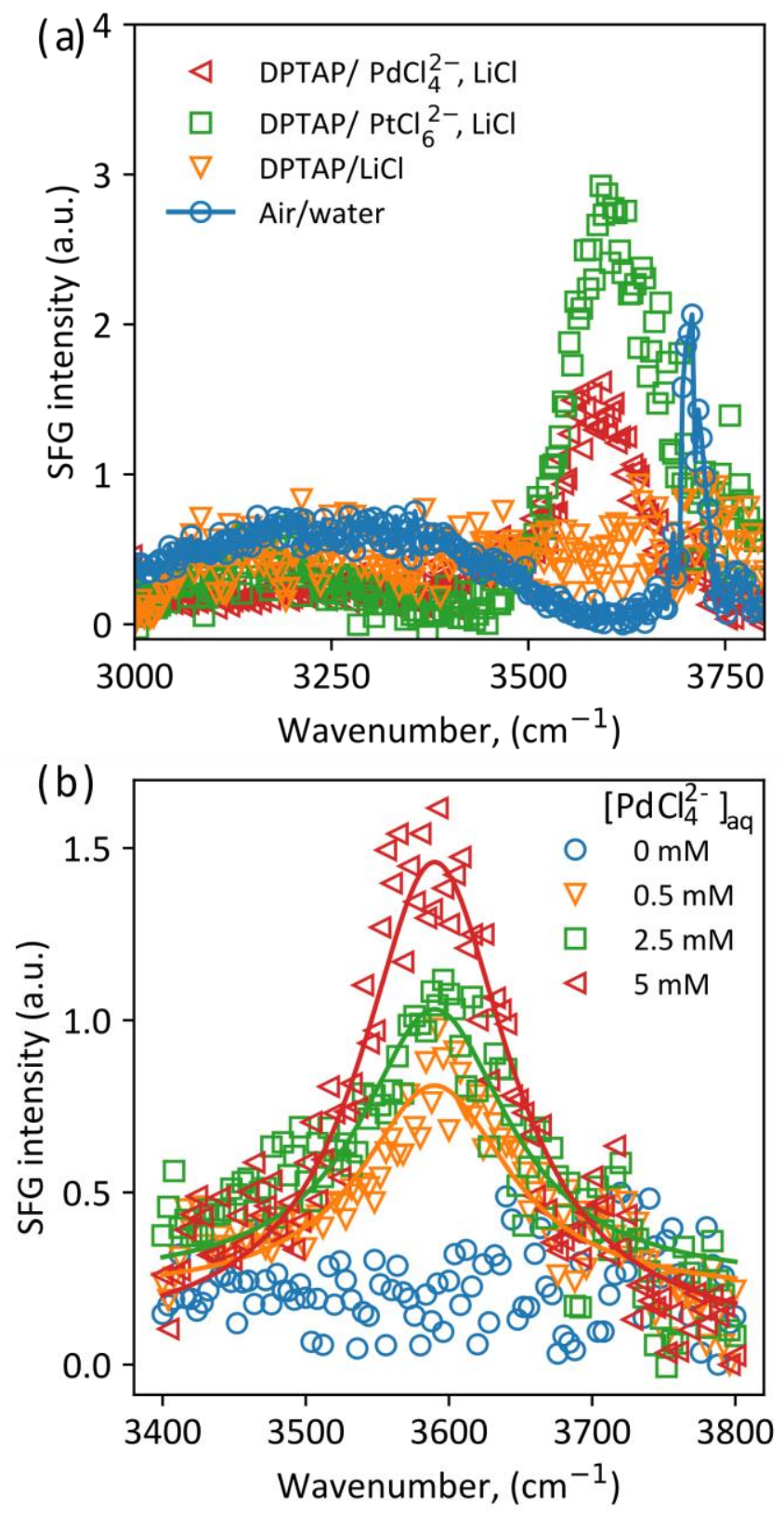

Figure 5. (a) VSFG spectra showing the -OH stretching modes of water. The symbols and colors correspond to different sample conditions as follows: red triangles - DPTAP spread on $5 \mathrm{mM} \mathrm{PdCl}_{4}{ }^{2-}$ in $0.5 \mathrm{M} \mathrm{LiCl}, \mathrm{pH}$ $=2$, green squares $-5 \mathrm{mM} \mathrm{PtCl}_{6}{ }^{2-}$ in $0.5 \mathrm{M} \mathrm{LiCl}, \mathrm{pH}=2$, orange inverted triangles - DPTAP spread on $0.5 \mathrm{M}$ $\mathrm{LiCl}, \mathrm{pH}=2$, and blue circles - air/water interface. (b) VSFG spectra showing the variation of the AIWHB peak with the concentration of $\mathrm{PdCl}_{4}{ }^{2-}$ in the subphase. Symbols represent experimental data whereas solid lines are fits to the experimental data obtained with equation 6 . All samples contain $0.5 \mathrm{M} \mathrm{LiCl}$ and are kept at $\mathrm{pH}=2$.

Figure $5 \mathrm{a}$ shows the VSFG spectra in the $-\mathrm{OH}$ stretch region for different aqueous subphase conditions. VSFG spectrum of the bare air/water interface, a well-studied reference, shows a peak at $3700 \mathrm{~cm}^{-1}$, 
corresponding to the dangling ("free") - $\mathrm{OH}$ of interfacial water. This peak is absent in the spectra obtained with a monolayer of DPTAP on $0.5 \mathrm{M} \mathrm{LiCl}(\mathrm{pH}=2)$ solution. Also, the "water-water hydrogen bond" (WWHB) band between $3200-3500 \mathrm{~cm}^{-1}$ drops significantly in strength. This is commensurate with effect of short Debye length due to the high ionic strength of the solution. The counterions screen the static electric field induced by the charged interface and disturb the orientational order of water molecules. In the presence of $5 \mathrm{mM} \mathrm{PtCl}_{6}{ }^{2-}$ or $\mathrm{PdCl}_{4}{ }^{2-}$ however, there is a clear peak appearing around $3600 \mathrm{~cm}^{-1}$. The peaks observed with metalates adsorbing to DPTAP are clearly different from the "free" $\mathrm{OH}$ peak. The peak observed with $\mathrm{PtCl}_{6}{ }^{2-}$ has been previously studied in detail and has been named as the anion-induced weak hydrogen bonded (AIWHB) water signal. ${ }^{13}$ One of the results from that study is presented here for comparison and contrast (Figure 5a, green squares). The proximity of the peak with $\mathrm{PdCl}_{4}{ }^{2-}$ to the AIWHB band induced by the adsorption of $\mathrm{PtCl}_{6}{ }^{2-}$ to DPTAP suggests that it also has a similar origin. The significantly lower intensity of this peak with $\mathrm{PdCl}_{4}{ }^{2-}$ in comparison to the $\mathrm{AIWHB}$ with $\mathrm{PtCl}_{6}{ }^{2-}$ is particularly interesting and will be discussed below. These results indicate that chlorometalates cause a unique hydrogen bonding environment near positively charged interfaces unlike simple ions such as chloride.

Figure $5 \mathbf{b}$ shows the variation of the AIWHB peak at different $\mathrm{PdCl}_{4}{ }^{2-}$ concentrations. In order to study the spectral features in more detail we have modeled the data using a Lorentzian as shown in Equation 6 . The fitted results are shown as solid lines in Figure $\mathbf{5 b}$ and the fit parameters are tabulated in Table S3. Unlike the two Lorentzian used to describe AIWHB obtained with $\mathrm{PtCl}_{6}{ }^{2-}$, the one with $\mathrm{PdCl}_{4}{ }^{2-}$ is modeled with a single peak centered at $3590 \mathrm{~cm}^{-1}$. Previously, the corresponding peak with $\mathrm{PtCl}_{6}{ }^{2-}$ (centered at $3606 \mathrm{~cm}^{-1}$ ) was attributed to AIWHB-down water molecules (first coordination shell of water molecules around $\mathrm{PtCl}_{6}{ }^{2-}$ that also interact with headgroup of DPTAP). The absence of corresponding further red-shifted peak (centered at $3534 \mathrm{~cm}^{-1}$ ) in $\mathrm{PdCl}_{4}{ }^{2-}$ and the lower intensity of the peak at $3590 \mathrm{~cm}^{-1}$ indicates that, although both metalates affect the hydrogen bonding environment, the hydration structures at the interface are different with them.

Sub-ensemble analysis. Sub-ensemble analysis of all-atom MD simulations of interfacial water can be used to understand origins of AIWHB water signal. Sub-ensemble analysis refers to the decomposition of all local environments about molecules/ions found within the ensemble distribution from the simulation trajectory. We note that the counterions of the metalates $\left(\mathrm{K}^{+}\right)$are not observed in the interfacial region in our simulations. Figure 6 shows the density-weighted orientation of water's OH dipoles near the interface. Water molecules at the interface are labeled AIWHB-Down, AIWHB-Up, WWHB-Down or WWHB-Up depending on the distance between water molecule, metal center and the $\mathrm{N}$-atoms on DPTAP (see Experimental Methods). Here WWHB refers to water-water hydrogen bond. These subgroups represent water molecules with similar hydrogen bonding environments.

AIWHB waters mainly coordinate chlorometalates. Therefore, their hydrogen bonding is weaker compared to water-water hydrogen bonding (appearing at $3200-3500 \mathrm{~cm}^{-1}$ ) which causes the VSFG signal from these molecules to appear at a correspondingly higher wavenumber region, around $3600 \mathrm{~cm}^{-1}$. AIWHB waters are divided into two subgroups. AIWHB-Up waters (orange curves in Figure 6 a and c, and indicated with yellow shade in $b$ and d) coordinate only chlorometalate and AIWHB-Down (yellow curves in Figure $6 a$ and $c$, and indicated with orange shade in b and d) waters are in the proximity of both chlorometalates and the DPTAP headgroup nitrogen. These two sub-ensembles were previously defined for $\mathrm{PtCl}_{6}{ }^{2-}$ as shown in Figure $\mathbf{6 a}$ and $\mathbf{6 b}$, and they successfully explain why the AIWHB band (Figure 5a, 
green squares) needs to be fit by two oppositely signed Lorentzian curves centered at $3534 \mathrm{~cm}^{-1}$ and 3606 $\mathrm{cm}^{-1}$ for WWHB-Up and WWHB-Down, respectively.
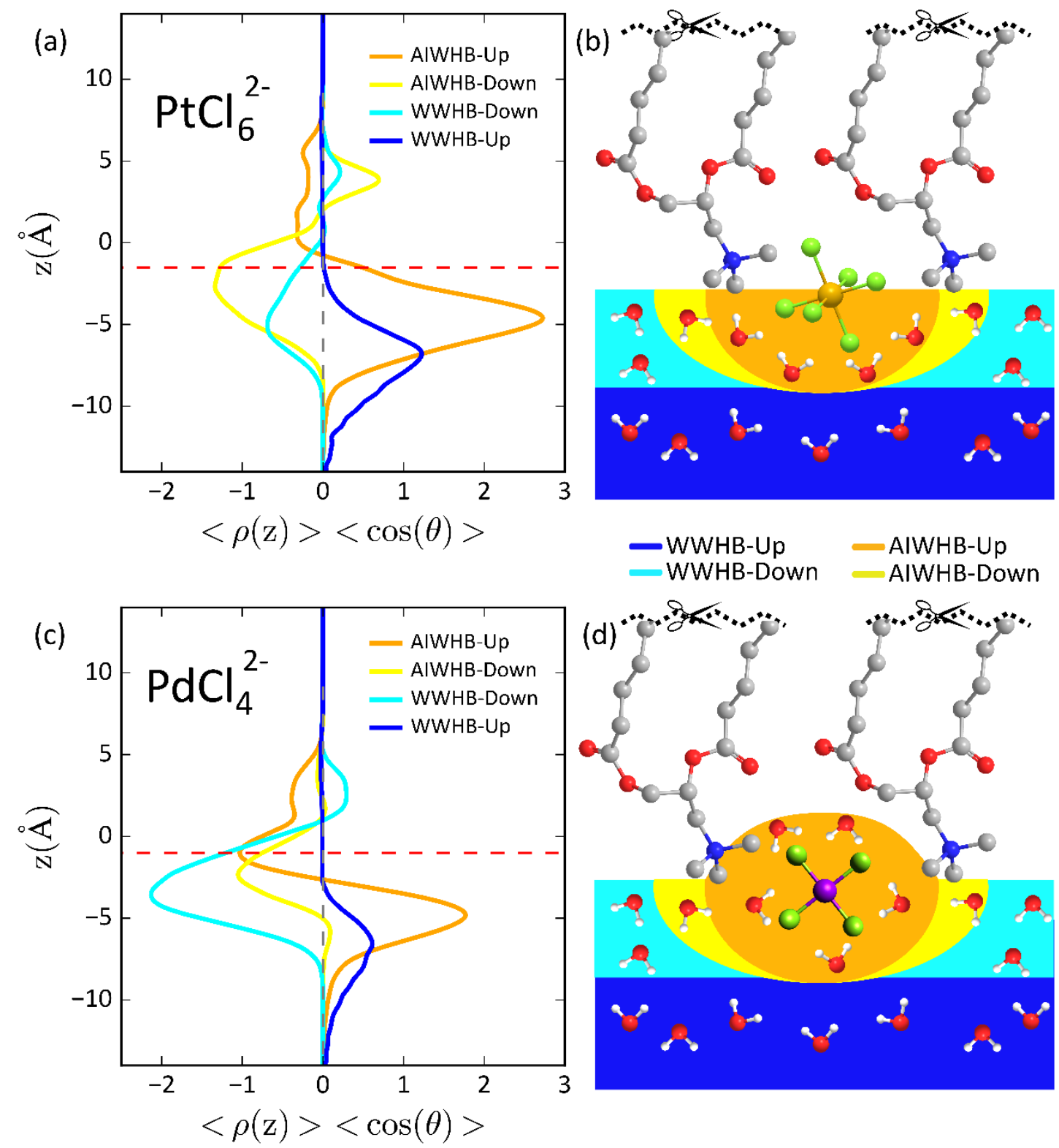

Figure 6. All-atom MD results showing the density-weighted orientation of different water populations interacting with (a) $\mathrm{PtCl}_{6}{ }^{2-}$ and (c) $\mathrm{PdCl}_{4}{ }^{2-}$ near the interface. Water molecules interacting with the complex near the interface are labeled as AIWHB-Up, AIWHB-Down, WWHB-Up, or WWHB-Down depending on the distance between water molecule $-\mathrm{Pd} / \mathrm{Pt}$ distance and water molecule $-\mathrm{N}$ (from DPTAP) distance. These labels correspond to the first coordination shell of water around the anionic complex away from (AIWHB-Up) and second coorination shell, towards the interface (AIWHB-Down), and second coordination shell of water underneath the chlorometalate, not interacting with DPTAP (WWHB-Up) and water molecules immedieately under DPTAP headgroups, not interacting with the chlorometalate (WWHB- 
Down). (b,d) show the schematic of different interfacial water populations in the presence of $\mathrm{PtCl}_{6}{ }^{2-}$ and $\mathrm{PdCl}_{4}{ }^{2-}$, respectively.

A similar sub-ensemble analysis for $\mathrm{PdCl}_{4}{ }^{2-}$ (Figure $6 \mathrm{c}$ ) exhibits significant differences. First, the AIWHB-Up (orange curve in Figure $6 \mathrm{c}$ ) waters have populations of almost equal up and down orientation, suggesting that the first hydration shell of $\mathrm{PdCl}_{4}{ }^{2-}$ stays more intact compared to $\mathrm{PtCl}_{6}{ }^{2-}$. Since AIWHB-Up have an almost equal number of water molecules pointing up and down (Figure $6 \mathrm{~d}$ ), they do not contribute to the VSFG signal. This explains the significant decrease in VSFG signal from the $\mathrm{PdCl}_{4}{ }^{2-}$ solution (Figure $\mathbf{5 a}$, red triangles). In contrast, the AIWHB-Down waters are preferentially oriented downward, suggesting that the observed small VSFG signal mainly originates from these water molecules, which corresponds to the AIWHB peak at $3590 \mathrm{~cm}^{-1}$.

Discussion. Long-range colloidal interactions have been suggested to play a role in determining selectivity of LLE in addition to the complexation between the extractant and the ion. ${ }^{34,81}$ Our results show that the converse need not always be true - even when the complexation is the same for two ions, a difference in organic phase structure at the mesoscale does not imply higher selectivity in LLE. A similar phenomenon is observed with lanthanide bearing $\mathrm{TOMA}-\mathrm{NO}_{3}$ complex fluids wherein the organic phase structure is not correlated with metal concentration. ${ }^{59}$ In the case of TOMA-SCN however, the organic phase structure is correlated with the metal concentration. ${ }^{59}$ These different anions affect the interfacial hydration structure around the extractant headgroup differently. ${ }^{74}$ These results indicate the importance of the water structure within the reverse micellar aggregates in determining the organic phase structure. Extraction of lanthanides with TOMA-NO $\mathrm{N}_{3}$ or TOMA-SCN is particularly interesting as lanthanides do not form anionic complexes in the aqueous phase at typical extraction conditions, unlike the chlorometalates presented here. ${ }^{53,82}$ The atypical selectivity of TOMA- $\mathrm{NO}_{3}$ towards light lanthanides is hypothesized to be due to the hydration properties of the lanthanides. ${ }^{59,83-84}$

The major difference in the effect of chlorometalates on the organic phase structure is the higher clustering of aggregates in the case of $\mathrm{PtCl}_{6}{ }^{2-}$ compared to that of $\mathrm{PdCl}_{4}{ }^{2-}$ (Figure 3). Given the similar metal content but different water content, these differences can be due to different geometries of $\mathrm{PtCl}_{6}{ }^{2-}$ and $\mathrm{PdCl}_{4}{ }_{4}^{2-}$ and different hydration structures in the reverse micellar aggregates. ${ }^{71}$ Differences in water and acid extraction between the two systems can also cause differences in aggregation structures. VSFG results show that the hydration structure of the chlorometalates, as they interact with the model extractant, are different. When $\mathrm{PtCl}_{6}{ }^{2-}$ adsorbs at the interface, it mostly loses the top half of the first hydration shell (Figure 6a, AIWHB-Up). In contrast, the first hydration shell of $\mathrm{PdCl}_{4}{ }^{2-}$ appears to have water molecules both above and below the chlorometalate. This correlates with the higher water content of the $\mathrm{PdCl}_{4}{ }^{2-}$ loaded organic phase compared to that for the $\mathrm{PtCl}_{6}{ }^{2-}$.

Unraveling the precise causal link between interfacial structure and bulk organic phase organization is challenging as a multitude of related factors affect the latter. The main difficulty in experimental studies is that the organic phase composition cannot be controlled component-wise. The present study reiterates this issue - even with equal concentration of one component (metalate) the organic phase structures are different. The difference between the metalates is primarily in their geometry which influences their hydration and possibly the co-extraction of water. We hypothesize that the interfacial hydration of the metalates is the link between organic phase structure and the geometry of the metalates. 
The formation of long-range structures has been attributed to the increased dispersion forces between the polar cores of the aggregates. ${ }^{19,24}$ Given that organic phase concentration of $\mathrm{PtCl}_{6}{ }^{2-}$ and $\mathrm{PdCl}_{4}{ }^{2-}$ are equal, the differences in their hydration environments could be the reason behind these differences. Higher number of chlorides present in $\mathrm{PtCl}_{6}{ }^{2-}$ could also enable higher interactions with water and other polar solutes, leading to larger structures. Alternatively, due to the higher loss of hydration shell in $\mathrm{PtCl}_{6}{ }^{2-}$ there might be a higher tendency for $\mathrm{PtCl}_{6}{ }^{2-}$ to associate with other polar molecules in the organic phase as a shielding mechanism. Another possibility is that the dehydrated $\mathrm{PtCl}_{6}{ }^{2-}$ has higher counterion condensation effect on the extractant and hence it enables lower curvature of the aggregate interface. This is commonly observed in classical micelle forming systems, where counterion condensation and dehydration are strongly related to micelle structure. ${ }^{85-89}$ Stronger counterion adsorption reduces the repulsion between the headgroups and leads to elongated structures. The arguments are not mutually exclusive but require a molecular level picture of the aggregates to definitively explain the origin of the differences in organic phase structure.

\section{CONCLUSIONS}

LLE is a very complex process with multiple factors governing the free energy landscape during the transfer of solutes between the aqueous and organic phases and the formation of nano- to meso-scale structures in the organic phase. Here, we isolated the effects of ion hydration and showed how it affects water extraction and the long range ordering in the organic phase. $\mathrm{PtCl}_{6}{ }^{2-}$ loses the top half of its hydration shell at the interface, which leads to significantly more clustering and dehydration of the organic phase. $\mathrm{PdCl}_{4}{ }^{2-}$, however, retains a relatively symmetrical hydration shell and shows relatively less clustering.

We have used multiple surface sensitive techniques, MD simulations, and bulk characterization experiments to identify the individual parts of this complex process. Some of the important conclusions would not be obtained without a combination of these techniques. For instance, the different VSFG signals in $\mathrm{PdCl}_{4}{ }^{2-}$ and $\mathrm{PtCl}_{6}{ }^{2-}$ systems could easily be interpreted as a difference in the amount of adsorbed metalates at the interface. However, XFNTR experiments and MD simulations clearly showed that their interfacial coverages were equal, providing strong evidence that the difference in AIWHB peaks were due to the different interfacial hydration. The sub-ensemble analysis of MD simulations provided a clear explanation of the different hydration structures. Karl Fischer analysis of the extracted organic phase showed that even though the metal concentrations are similar, water content depends on the identity of the metalate. Finally, all the information obtained from the interfacial studies correlated with the different clustering behaviors in the organic phase and different water extraction, providing a connection between the amphiphile-metalate interactions at the interface and in the organic phase. The relationship between ion hydration and heterogeneous microstructure of the organic phase presented here are relevant to the broader field of ionic liquids. ${ }^{90-93}$

LLE is typically driven by a few $\mathrm{kT}$ difference in the free energy of the organic and aqueous phase structures as multiple interactions with similar magnitudes compete. Understanding the molecular scale mechanisms in detail will provide big opportunities in developing more efficient, more robust, and less energy intensive processes.

\section{EXPERIMENTAL AND COMPUTATIONAL METHODS}


Materials. Methyltrioctylammonium chloride (TOMA.Cl), hexachloroplatinic acid $\left(\mathrm{H}_{2} \mathrm{PtCl}_{6}, 8 \mathrm{wt} \%\right.$ aqueous solution), potassium chloropalladate $\left(\mathrm{K}_{2} \mathrm{PdCl}_{4}\right)$, chloroform (anhydrous, $\geq 99 \%$ pure) were purchased from Sigma-Aldrich. 1,2-dipalmitoyl-3-trimethylammonium-propane (DPTAP) was purchased from Avanti Polar Lipids. All the chemicals were used as received without further purification.

Liquid-Liquid Extraction. Aqueous solutions of $\mathrm{H}_{2} \mathrm{PtCl}_{6}$ and $\mathrm{K}_{2} \mathrm{PdCl}_{4}$ with $\mathrm{HCl}$ were prepared by dissolving the corresponding masses of salts in volumetric flasks. The extractant phase was prepared by dissolving TOMA.Cl in toluene to obtain a $0.2 \mathrm{M}$ solution, and pre-equilibrating the solution with $5 \mathrm{M} \mathrm{HCl}$ for one hour. Equal volumes of the organic phase and the aqueous phases were contacted in a glass vial by vortex mixing for 1 minute, followed by radial shaking for one hour at room temperature. The phases were then separated by centrifugation and stored separately in glass vials.

SAXS Experiments. SAXS measurements were conducted at sector 12-ID-C of Advanced Photon Source, Argonne National Laboratory. $X$-ray energy was $18 \mathrm{keV}(\lambda=0.516 \AA)$. Samples were contained in $2 \mathrm{~mm}$ OD quartz capillaries. Standard data reduction procedures were used to obtain the 1D scattering profiles for the samples. Toluene was used as a standard for absolute intensity calibration. SAXS data was analyzed as described previously. Briefly, background subtracted (quartz capillary and toluene) and calibrated SAXS data was fit to a model of clustering hard-spheres.

We model the aggregates formed by extractants as core-shell structures. Due to the high extractant concentration present in our system, the inter-aggregate interactions cannot be ignored. Specifically, with increasing presence of polar solutes (water or ions) in the core of the aggregates, there can be attractive interactions between them that lead to the formation of larger scale structures, referred as clusters (Figure 1). So, we include a term in the structure factor that accounts for the attraction-induced clustering of the aggregates. We use hard-sphere structure factor $\left(S_{p}(Q)\right)$ based on Percus-Yevick approximation and include a clustering term $\left(P_{c}(Q)\right)$ that arises due to weak attraction between the aggregates. ${ }^{68}$

$I_{\text {measured }}=$ Calibration $* I(Q)+$ Background (1)

$I(Q)=P(Q) S(Q)$

$P(Q)=\frac{\phi_{p}}{V_{s}}\left[3 V_{c}\left(\rho_{c}-\rho_{s}\right) j\left(Q R_{c}\right)+3 V_{s}\left(\rho_{s}-\rho_{\text {solvent }}\right) j\left(Q R_{s}\right)\right]^{2}$

$j(x)=\frac{\sin x-x \cos x}{x^{3}}$

$S(Q)=f P_{c}(Q)+S_{i}(Q)$

In the above equations $I(Q)$ is the absolute intensity (scattering cross section) in $\mathrm{cm}^{-1} ; P(Q)$ is the form factor of scatterers; $S(Q)$ is the total structure factor; $\varphi_{\mathrm{p}}$ is the volume fraction of the scatterers; $R_{c}$ and $R_{s}$ are core and shell radius respectively; $V_{c}$ and $V_{s}$ are volumes of core and shell respectively; $\rho_{c}, \rho_{s,}$ and $\rho_{\text {solvent }}$ are scattering length densities of core, shell, and solvent respectively. In the structure factor equation, $f+1$ is the number of aggregates per cluster, $P_{c}$ is the form factor of the cluster, and $S_{p}$ is the hard-sphere structure factor which is a function of the hard-sphere volume fraction $\left(\phi_{H S}\right)$ and size (same as the size of the shell). Form factor of the cluster is obtained using the Guinier-Porod equation. This theoretical curve is fit to the experimental data to obtain optimal fit values for $R_{c}, R_{s}, \rho_{c} \rho_{s,} \phi_{H S}, f, R_{g}$ (radius of gyration of the cluster) and $d$ (Porod exponent of the cluster). 
ICP-MS analysis. Aqueous phase compositions, before and after LLE, were determined using ICP-MS. Aliquots of the aqueous phases were diluted with a $2 \mathrm{wt} \% \mathrm{HNO}_{3}+2 \mathrm{wt} \% \mathrm{HCl}$ solution (both acids of trace metal grade). Quantitative standards were made using a $1000 \mu \mathrm{g} / \mathrm{mL}$ Pt elemental standard and a 10,000 $\mathrm{ug} / \mathrm{mL}$ Pd elemental standard (Inorganic Ventures, Christiansburg, VA, USA) which was used to create a $1000 \mathrm{ng} / \mathrm{mL}$ mixed element standard in 3.0\% nitric acid (v/v) in a total sample volume of $50 \mathrm{~mL}$.

ICP-MS was performed on a computer-controlled (QTEGRA software) Thermo iCapQ ICP-MS (Thermo Fisher Scientific, Waltham, MA, USA) operating in STD mode and equipped with an ESI SC-2DX PrepFAST autosampler (Omaha, NE, USA). Internal standard was added inline using the prepFAST system and consisted of $1 \mathrm{ng} / \mathrm{mL}$ of a mixed element solution containing $\mathrm{Bi}, \mathrm{In},{ }^{6} \mathrm{Li}, \mathrm{Sc}, \mathrm{Tb}, \mathrm{Y}$ (IV-ICPMS-71D from Inorganic Ventures). Online dilution was also carried out by the prepFAST system and used to generate calibration curves consisting of 1000, 500, 250, 50 and $10 \mathrm{ng} / \mathrm{g}$ Pt and Pd. Each sample was acquired using 1 survey run (10 sweeps) and 3 main (peak jumping) runs ( 40 sweeps). The isotopes selected for analysis were ${ }^{105} \mathrm{Pd},{ }^{106} \mathrm{Pd},{ }^{108} \mathrm{Pd},{ }^{195} \mathrm{Pt},{ }^{196} \mathrm{Pt},{ }^{198} \mathrm{Pt}$, and ${ }^{89} \mathrm{Y},{ }^{115} \mathrm{In},{ }^{159} \mathrm{~Tb}$ (chosen as internal standards for data interpolation and machine stability). Instrument performance is optimized daily through autotuning followed by verification via a performance report (passing manufacturer specifications).

Karl Fischer Analysis. Water content of samples was analyzed with 831 KF Coulometer/860 KF Thermoprep, Metrohm. Hydranal Coulomat E (Honeywell Fluka) was used as the titration reagent. Approximately $0.2 \mathrm{~g}$ of the samples was heated to $150^{\circ} \mathrm{C}$ in the oven and the evaporated moisture was carried into titration vessel by pumped air ( $60 \mathrm{~mL} / \mathrm{min})$. Titration was carried out for a minimum of $500 \mathrm{~s}$. Blanks were measured with sealed $6 \mathrm{~mL}$ glass vials. All samples were measured 7 times. Weight fractions obtained by Karl Fischer analysis, after subtracting the blank, were converted to molarities using the measured density of samples.

Interfacial Experiments. 1,2-dipalmitoyl-3-trimethylammonium-propane, chloride salt (DPTAP) was used as a Langmuir monolayer forming surfactant analogue to TOMA.Cl. Both have quaternary ammonium headgroup but different tail structures. Although DPTAP is not used as an extractant industrially, its ability to form a crystalline monolayer enables clear analysis of interfacial changes that accompany extraction. Preparation of these monolayers for XFNTR and VSFG measurements has been described earlier. ${ }^{13,74}$ Briefly, for XFNTR measurements, $200 \mathrm{~mL}$ of the aqueous subphase $(0.5 \mathrm{M} \mathrm{LiCl}, \mathrm{pH}=2$ with different metalate concentrations) is poured into the PTFE Langmuir trough. The surface tension is monitored while the surface is aspirated to remove any floating contaminants. Approximately $100 \mu \mathrm{L}$ of $0.25 \mathrm{mM}$ DPTAP solution in chloroform is spread on the surface by dropwise addition using a Hamilton glass syringe. The barrier is then closed at $8 \mathrm{~cm}^{2} / \mathrm{min}$ until the surface pressure reaches $10 \mathrm{mN} / \mathrm{m}$.

VSFG Measurement. The VSFG measurements are acquired using an EKSPLA system, which has been described previously. ${ }^{13,74}$ Briefly, the setup consists of a picosecond laser system (PL223150-50), a harmonic unit (SFGH500-H/2H), an optical parametric generator with difference frequency generation (PG501-DFG1P), a spectrometer and a photomultiplier tube detector (Hamamatsu, R7899) connected to a monochromator (Sol, MS2001). An amplified Nd:YAG laser system produces $28 \mathrm{~mJ}, 29$ ps pulses centered at $1064 \mathrm{~nm}$ with a repetition rate of $50 \mathrm{~Hz}$. The harmonic unit splits the $1064 \mathrm{~nm}$ laser and a portion is passed through a second harmonic crystal to generate two beams of $532 \mathrm{~nm}$. One of the $532 \mathrm{~nm}$ beams and the $1064 \mathrm{~nm}$ beam are used to generate a narrowband IR pulse tunable from $1000-4000 \mathrm{~cm}^{-1}$ via optical parametric generator and difference frequency generation. Other $532 \mathrm{~nm}$ laser beam which passes 
through an adjustable delay stage is overlapped spatially and temporarily with an IR beam to generate a sum frequency (SF) signal. The polarization of $532 \mathrm{~nm}$ beam is adjusted with the help of $\lambda / 2$ waveplate, whereas the IR polarization is adjusted by using a computer-controlled motorized mirrors. The VSFG signal is selected using a Glan polarizer. The SFG signal is then directed to a monochromator and collected with a photomultiplier tube.

The VSFG spectrometer employs the reflection geometry where the incident angles of the visible and IR beams are $60^{\circ}$ and $55^{\circ}$ respectively to the surface normal. The visible and IR laser beams are attenuated to an average energy of 800 and $65 \mu \mathrm{J}$ respectively. A motorized piezoelectric rotation stage is used to rotate the sample to avoid beam damage. Each spectrum is collected with a $4 \mathrm{~cm}^{-1}$ increment over the range of $2800-3800 \mathrm{~cm}^{-1}$ and averaged 300 laser shots per point. The spectra are normalized against the SFG spectrum of z-cut quartz.

VSFG Data Global Fit. The anion-induced weakly hydrogen-bonded (AIWHB) band centered around 3600 $\mathrm{cm}^{-1}$ to Equation $6,{ }^{94}$ with a single resonance for the $\mathrm{PdCl}_{4}{ }^{2-}$ solution unlike $\mathrm{PtCl}_{6}{ }^{2-}$ which requires two resonant peaks. ${ }^{13}$ This suggests that mainly one type of water hydrogen bonding environment contributes strongly to this band. Therefore, we used a single Lorentzian

$I_{V S F G} \propto\left|A_{N R} e^{i \emptyset_{N R}}+\frac{A_{1 n}}{\omega-\omega_{1}-i \Gamma_{1}}\right|^{2}$

where, $A_{N R}$ and $e^{i \emptyset_{N R}}$ are the non-resonant amplitude and phase, $\omega$ and $\omega_{1}$ are the tunable IR and the resonance frequency, and $\Gamma_{1}$ and $A_{1 n}$ are the peak width and amplitude.

XFNTR Experiments. XFNTR experiments were conducted at sector 15-ID-C of Advanced Photon Source at the Argonne National Laboratory using the experimental setup described elsewhere. ${ }^{14} \mathrm{X}$-ray energy was $17 \mathrm{keV}$. Fluorescence photons emanating from the sample, perpendicular to the interface, were collected as a function of the incidence angle, using an energy dispersive Vortex detector. The integrated emission in the L-alpha emission region of $\mathrm{Pt}$, as a function of the vertical momentum transfer $\left(q_{z}\right)$ was modeled considering the experimental geometry and the atomic spectral properties of $\mathrm{Pt}^{17}$

MD simulations. All MD simulations were performed using the GROMACS 2016.2 software package ${ }^{95}$ to study the water solvation of the heavy anionic complex at the charged liquid surface. The simulation box was built with the aqueous phase that is sandwiched between two DPTAP surfactant monolayers and a large empty region beyond the DPTAP monolayers. Periodic boundary conditions were applied in all three dimensions. The modeled aqueous solution contains $0.5 \mathrm{M} \mathrm{LiCl}, 0.01 \mathrm{M} \mathrm{HCl}(\mathrm{pH}=2)$, and $0.5 \mathrm{M} \mathrm{PtCl}_{6}{ }^{2-}$ or $\mathrm{PdCl}_{4}{ }^{2-}$ with $\mathrm{K}^{+}$as the counterion with a goal to reproduce the highest concentrations experimentally explored. The DPTAP surfactant was positively charged at its head group $\left(-\mathrm{NH}_{3}{ }^{+}\right)$, which was charge compensated with $\mathrm{Cl}^{-}$. The CHARMM force field for DPTAP ${ }^{96}$ and all ions, ${ }^{97}$ and the TIP3P force field ${ }^{98}$ for water were employed in the simulation. The energy minimized configuration was equilibrated in the isothermal-isobaric (NPT) ensemble for 200 ns, where the Nose-Hoover thermostat/Parrinello-Rahman barostat ${ }^{99-100}$ with temperature/pressure coupling time constant of 2/0.4 ps were chosen. Following the NPT equilibration, a 100 ns trajectory was obtained in the canonical (NVT) ensemble with a 10 ps sampling time as the production runs for the following analyses. All simulations adopt a $1.2 \mathrm{~nm}$ Lennard-Jones and short-range Coulombic interaction cutoff. Long-range electrostatics were computed using the ParticleMesh Ewald summation. ${ }^{101}$ The time step is $2 \mathrm{fs}$. 

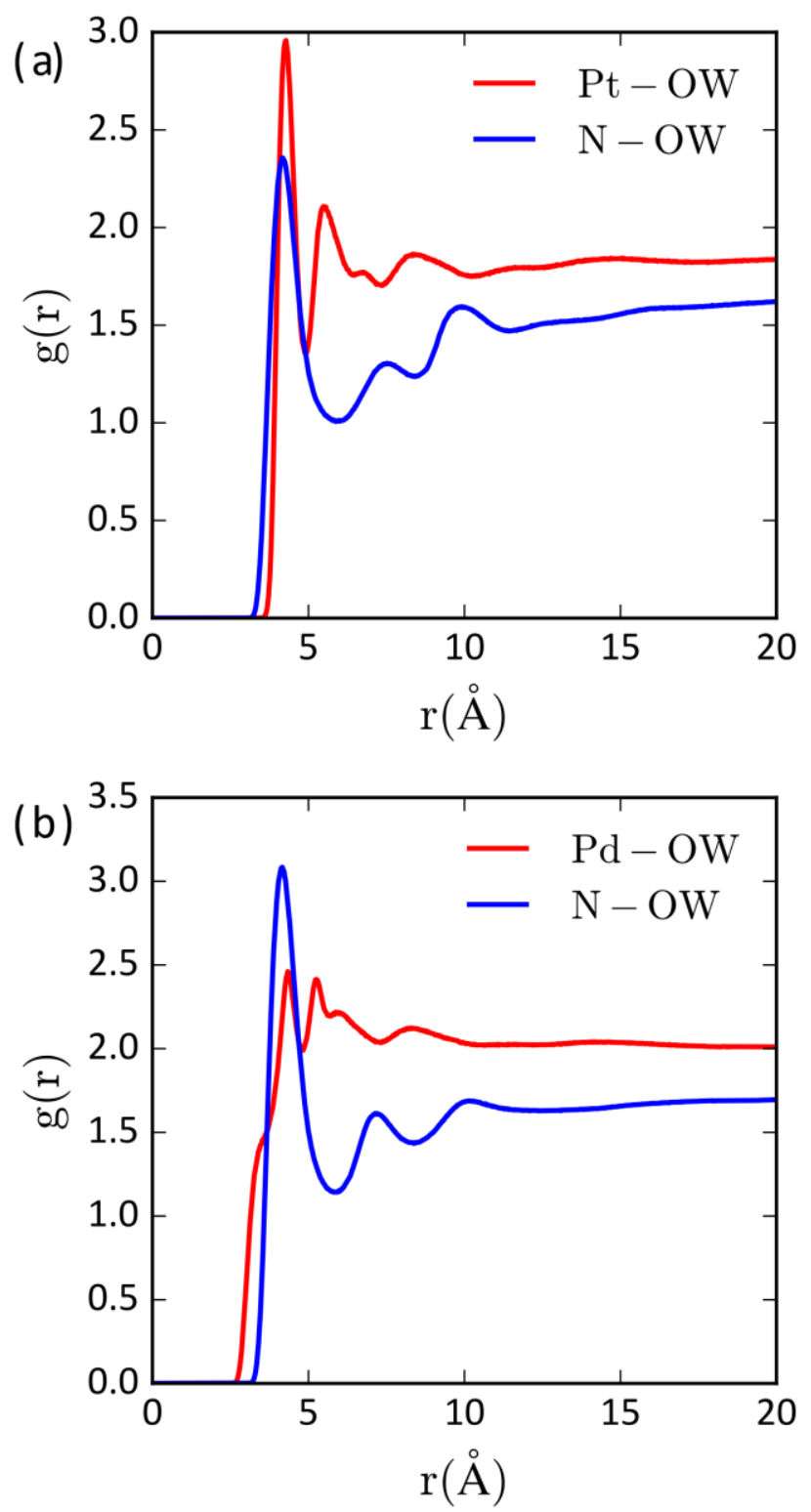

Figure 7 (a) RDF between the Pt atom and water oxygen atom (Ow, red curve) and RDF between the DPTAP head group ( $\mathrm{N}$ atom) and $\mathrm{OW}$ atom (blue curve) for the $\mathrm{PtCl}_{6}{ }^{2-}$ interface system. The corresponding $\mathrm{Pd}-\mathrm{OW}$ and $\mathrm{N}-\mathrm{OW} \mathrm{RDFs}$ for the $\mathrm{PdCl}_{4}{ }^{2-}$ interface system are shown in (b).

Figure 7 (a) and (b) show the radial distribution functions in the simulated $\mathrm{PtCl}_{6}{ }^{2-}$ (a) and $\mathrm{PdCl}_{4}{ }^{2-}$ (b) interfacial systems, respectively. Based on the Pt-Ow and Pd-Ow RDFs, we estimated the first minima as a distance criteria for determining the first solvation shell of the $\mathrm{PtCl}_{6}{ }^{2-}$ or $\mathrm{PdCl}_{4}{ }^{2-}$ anion. Similarly, the second minima define cutoff distances corresponding to the respective second solvation shell. Based on the N-Ow RDFs, the solvation shell of DPTAP head group are considered to be truncated at the respective first minimum.

Table 1: Descriptions of different interfacial water populations and their cutoff distances chosen based on the corresponding RDF in Figure 7. 


\begin{tabular}{|c|c|c|c|c|}
\hline System & AIWHB-UP & AIWHB-DOWN & WWHB-DOWN & WWHB-UP \\
\hline Description & $\begin{array}{l}\text { water molecules } \\
\text { in the first } \\
\text { solvation shell of } \\
\mathrm{PtCl}_{6}{ }^{2-} \text { or } \mathrm{PdCl}_{4}{ }^{2-}\end{array}$ & $\begin{array}{l}\text { water molecules in the } \\
\text { second solvation shell of } \\
\mathrm{PtCl}_{6}{ }^{2-} \text { or } \mathrm{PdCl}_{4}{ }^{2-} \text { and in } \\
\text { the solvation shell of } \\
\text { DPTAP head group }\end{array}$ & $\begin{array}{l}\text { water molecules } \\
\text { outside the } \\
\text { second solvation } \\
\text { shell of } \mathrm{PtCl}_{6}^{2-} \text { or } \\
\mathrm{PdCl}_{4}^{2-} \text { but within } \\
\text { the solvation shell } \\
\text { of DPTAP head } \\
\text { group }\end{array}$ & $\begin{array}{l}\text { water molecules } \\
\text { outside the first } \\
\text { solvation shell of } \\
\mathrm{PtCl}_{6}{ }^{2-} \text { or } \mathrm{PdCl}_{4}{ }^{2-} \\
\text { and outside the } \\
\text { solvation shell of } \\
\text { DPTAP head group }\end{array}$ \\
\hline $\mathrm{PtCl}_{6}^{2-}$ & $r_{\text {Ow...Pt }}<5.0 \AA$ & $\begin{array}{l}5.0<r_{\text {Ow...Pt }}<6.5 \AA, \\
r_{\text {Ow...N }}<6.0 \AA\end{array}$ & $\begin{array}{l}r_{\text {Ow...Pt }}>6.5 \AA, \\
r_{\text {Ow...N }}<6.0 \AA\end{array}$ & $\begin{array}{l}r_{\text {Ow...Pt }}>5.0 \AA, \\
r_{\text {Ow...N }}>6.0 \AA\end{array}$ \\
\hline $\mathrm{PdCl}_{4}^{2-}$ & $r_{\text {Ow...Pd }}<4.8 \AA$ & $\begin{array}{l}4.8<r_{\text {Ow...Pd }}<5.7 \AA \text {, } \\
r_{\text {OW...N }}<5.8 \AA\end{array}$ & $\begin{array}{l}r_{\text {Ow...Pd }}>5.7 \AA, \\
r_{\text {Ow... }}<5.8 \AA\end{array}$ & $\begin{array}{l}r_{\text {Ow...Pd }}>4.8 \AA, \\
r_{\text {Ow...N }}>5.8 \AA\end{array}$ \\
\hline
\end{tabular}

\section{ASSOCIATED CONTENT}

\section{SUPPORTING INFORMATION.}

The supporting information consists of: tables providing the ICP-MS and Karl Fischer analysis results, fit parameters for SAXS profiles, discussion on alternative SAXS models, form factor and structure factor decomposition using the cluster model, discussion on the effect of $\mathrm{HCl}$ versus $\mathrm{LiCl}$ media for extraction, discussion on acid extraction with TOMA, X-ray fluorescence near total reflection results for metalate adsorption on DPTAP monolayers and supporting MD simulations, fit parameters for VSFG spectra, and the VSFG spectra in the methyl stretch region for DPTAP monolayers with metalate adsorption.

\section{AUTHOR INFORMATION}

\section{Corresponding Author}

*Email: ahmet@anl.gov. Web: www.anl.gov/profile/ahmet-uysal

\section{Author Contributions}

The manuscript was written through contributions of all authors. All authors have given approval to the final version of the manuscript.

Notes

Authors declare no competing financial interest.

\section{ACKNOWLEDGEMENTS}

We thank Soenke Seifert and Wei Bu for their help in conducting SAXS and XFNTR experiments, respectively. We thank Ross Ellis and Kirstian MacRuary for some early ideas on this system. The work presented here is supported by the U.S. Department of Energy, Office of Basic Energy Science, Division of Chemical Sciences, Geosciences, and Biosciences, under contract DE-AC02-06CH11357. Z.L. and A.E.C. are supported by the same program, under contract DE-SC0001815. Use of the Advanced Photon Source, an Office of Science User Facility operated for the U.S. Department of Energy (DOE) Office of Science by Argonne National Laboratory, was supported by the U.S. DOE under Contract No. DE-AC02-06CH11357. 21 
NSF's ChemMatCARS Sector 15 is principally supported by the Divisions of Chemistry (CHE) and Materials Research (DMR), National Science Foundation, under Grant NSF/CHE-1834750. MD simulations were carried out at the computing resources provided on Blues, a high-performance computing cluster operated by the Laboratory Computing Resource Center at Argonne National Laboratory. ICP-MS analysis was performed at the Northwestern University Quantitative Bio-element Imaging Center supported by NASA Ames Research Center Grant NNA04CC36G.

\section{ABBREVIATIONS}

SAXS, small angle X-ray scattering; TOMA, methyltrioctylammonium; XFNTR, X-ray fluorescence near total reflection; VSFG, vibrational sum frequency generation; LLE, liquid-liquid extraction

\section{REFERENCES}

1. Sholl, D. S.; Lively, R. P., Seven Chemical Separations to Change the World. Nature 2016, 532, 435-437.

2. National Academies of Sciences, E.; Medicine, A Research Agenda for Transforming Separation Science. The National Academies Press: Washington, DC, 2019.

3. Moyer, B., Changing the Landscape in Solvent Extraction. 1st ed.; CRC Press: Boca Raton, 2020; Vol. 23, p 310.

4. $\quad$ Perreault, L. L.; Giret, S.; Gagnon, M.; Florek, J.; Lariviere, D.; Kleitz, F., Functionalization of Mesoporous Carbon Materials for Selective Separation of Lanthanides under Acidic Conditions. ACS Appl. Mater. Interfaces 2017, 9, 12003-12012.

5. Budnyak, T. M.; Gladysz-Plaska, A.; Strizhak, A. V.; Sternik, D.; Komarov, I. V.; Majdan, M.; Tertykh, V. A., Imidazole-2yl-Phosphonic Acid Derivative Grafted onto Mesoporous Silica Surface as a Novel Highly Effective Sorbent for Uranium(VI) Ion Extraction. ACS Appl. Mater. Interfaces 2018, 10, 6681-6693.

6. Daliran, S.; Miri, M. G.; Oveisi, A. R.; Khajeh, M.; Navalon, S.; Alvaro, M.; Ghaffari-Moghaddam, M.; Delarami, H. S.; Garcia, H., A Pyridyltriazol Functionalized Zirconium Metal-Organic Framework for Selective and Highly Efficient Adsorption of Palladium. ACS Appl. Mater. Interfaces 2020, 12, 2522125232.

7. Zhang, Z. H.; Lan, J. H.; Yuan, L. Y.; Sheng, P. P.; He, M. Y.; Zheng, L. R.; Chen, Q.; Chai, Z. F.; Gibson, J. K.; Shi, W. Q., Rational Construction of Porous Metal-Organic Frameworks for Uranium(VI) Extraction: The Strong Periodic Tendency with a Metal Node. ACS Appl. Mater. Interfaces 2020, 12, 14087-14094.

8. Ambaye, T. G.; Vaccari, M.; Castro, F. D.; Prasad, S.; Rtimi, S., Emerging Technologies for the Recovery of Rare Earth Elements (REEs) from the End-of-life Electronic Wastes: A Review on Progress, Challenges, and Perspectives. Environ. Sci. Pollut. Res. 2020, 27, 36052-36074.

9. Laxman, K.; Myint, M. T.; Bourdoucen, H.; Dutta, J., Enhancement in Ion Adsorption Rate and Desalination Efficiency in a Capacitive Deionization Cell through Improved Electric Field Distribution Using Electrodes Composed of Activated Carbon Cloth Coated with Zinc Oxide Nanorods. ACS Appl. Mater. Interfaces 2014, 6, 10113-20.

10. Tiwari, B.; Zhang, D.; Winslow, D.; Lee, C. H.; Hao, B.; Yap, Y. K., A Simple and Universal Technique To Extract One- and Two-Dimensional Nanomaterials from Contaminated Water. ACS Appl. Mater. Interfaces 2015, 7, 26108-16.

11. Balaram, V., Rare Earth Elements: A review of Applications, Occurrence, Exploration, Analysis, Recycling, and Environmental Impact. Geosci. Front. 2019, 10, 1285-1303. 
12. Rock, W.; Oruc, M. E.; Ellis, R. J.; Uysal, A., Molecular Scale Description of Anion Competition on Amine-Functionalized Surfaces. Langmuir 2016, 32, 11532-11539.

13. Rock, W.; Qiao, B. F.; Zhou, T. C.; Clark, A. E.; Uysal, A., Heavy Anionic Complex Creates a Unique Water Structure at a Soft Charged Interface. J. Phys. Chem. C 2018, 122, 29228-29236.

14. Uysal, A.; Rock, W.; Qiao, B. F.; Bu, W.; Lin, B. H., Two-Step Adsorption of PtCl62- Complexes at a Charged Langmuir Monolayer: Role of Hydration and Ion Correlations. J. Phys. Chem. C 2017, 121, 25377-25383.

15. Servis, M. J.; Clark, A. E., Surfactant-enhanced Heterogeneity of the Aqueous Interface Drives Water Extraction into Organic Solvents. Phys. Chem. Chem. Phys. 2019, 21, 2866-2874.

16. Bera, M. K.; Luo, G. M.; Schlossman, M. L.; Soderholm, L.; Lee, S.; Antonio, M. R., Erbium(III) Coordination at the Surface of an Aqueous Electrolyte. J. Phys. Chem. B 2015, 119, 8734-8745.

17. Bu, W.; Mihaylov, M.; Amoanu, D.; Lin, B. H.; Meron, M.; Kuzmenko, I.; Soderholm, L.; Schlossman, M. L., X-ray Studies of Interfacial Strontium-Extractant Complexes in a Model Solvent Extraction System. J. Phys. Chem. B 2014, 118, 12486-12500.

18. Liang, Z.; Bu, W.; Schweighofer, K. J.; Walwark, D. J.; Harvey, J. S.; Hanlon, G. R.; Amoanu, D.; Erol, C.; Benjamin, I.; Schlossman, M. L., Nanoscale View of Assisted Ion Transport Across the LiquidLiquid Interface. P. Natl. Acad. Sci. U.S.A 2019, 116, 18227-18232.

19. Erlinger, C.; Belloni, L.; Zemb, T.; Madic, C., Attractive Interactions Between Reverse Aggregates and Phase Separation in Concentrated Malonamide Extractant Solutions. Langmuir 1999, 15, 2290-2300.

20. Zemb, T.; Bauer, C.; Bauduin, P.; Belloni, L.; Déjugnat, C.; Diat, O.; Dubois, V.; Dufrêche, J.-F.; Dourdain, S.; Duvail, M.; Larpent, C.; Testard, F.; Pellet-Rostaing, S., Recycling Metals by Controlled Transfer of Ionic Species Between Complex Fluids: en Route to "Ienaics". Colloid Polym. Sci. 2015, 293, 122.

21. Baldwin, A. G.; Servis, M. J.; Yang, Y.; Bridges, N. J.; Wu, D. T.; Shafer, J. C., The Structure of Tributyl Phosphate Solutions: Nitric Acid, Uranium (VI), and Zirconium (IV). J. Mol. Liq. 2017, 246, 225235.

22. Servis, M. J.; Piechowicz, M.; Shkrob, I. A.; Soderholm, L.; Clark, A. E., Amphiphile Organization in Organic Solutions: An Alternative Explanation for Small-Angle X-ray Scattering Features in Malonamide/Alkane Mixtures. J. Phys. Chem. B 2020, 124, 10822-10831.

23. Ellis, R. J.; Audras, M.; Antonio, M. R., Mesoscopic Aspects of Phase Transitions in a Solvent Extraction System. Langmuir 2012, 28, 15498-15504.

24. Ellis, R. J.; Meridiano, Y.; Muller, J.; Berthon, L.; Guilbaud, P.; Zorz, N.; Antonio, M. R.; Demars, T.; Zemb, T., Complexation-induced Supramolecular Assembly Drives Metal-ion extraction. Chem. Eur. J. 2014, 20, 12796-807.

25. Spadina, M.; Bohinc, K.; Zemb, T.; Dufrache, J. F., Colloidal Model for the Prediction of the Extraction of Rare Earths Assisted by the Acidic Extractant. Langmuir 2019, 35, 3215-3230.

26. Spadina, M.; Bohinc, K.; Zemb, T.; Dufreche, J. F., Synergistic Solvent Extraction Is Driven by Entropy. ACS Nano 2019, 13, 13745-13758.

27. Chiarizia, R.; Nash, K. L.; Jensen, M. P.; Thiyagarajan, P.; Littrell, K. C., Application of the Baxter Model for Hard Spheres with Surface Adhesion to SANS data for the U(VI)-HNO3, TBP-n-dodecane System. Langmuir 2003, 19, 9592-9599.

28. Chiarizia, R.; Jensen, M. P.; Borkowski, M.; Ferraro, J. R.; Thiyagarajan, P.; Littrell, K. C., Third Phase Formation Revisited: The U(VI), HNO3-TBP, n-Dodecane System. Solvent Extr. Ion Exc. 2003, 21, 127.

29. Ellis, R. J.; Antonio, M. R., Coordination Structures and Supramolecular Architectures in a Cerium(III)-Malonamide Solvent Extraction System. Langmuir 2012, 28, 5987-5998. 
30. Mu, J.; Motokawa, R.; Akutsu, K.; Nishitsuji, S.; Masters, A. J., A Novel Microemulsion Phase Transition: Toward the Elucidation of Third-Phase Formation in Spent Nuclear Fuel Reprocessing. J. Phys. Chem. B 2018, 122, 1439-1452.

31. Motokawa, R.; Kobayashi, T.; Endo, H.; Mu, J.; Williams, C. D.; Masters, A. J.; Antonio, M. R.; Heller, W. T.; Nagao, M., A Telescoping View of Solute Architectures in a Complex Fluid System. ACS Cent. Sci. 2019, 5, 85-96.

32. Ellis, R. J.; Meridiano, Y.; Chiarizia, R.; Berthon, L.; Muller, J.; Couston, L.; Antonio, M. R., Periodic Behavior of Lanthanide Coordination within Reverse Micelles. Chem. Eur. J. 2013, 19, 2663-2675.

33. Baldwin, A. G.; Ivanov, A. S.; Williams, N. J.; Ellis, R. J.; Moyer, B. A.; Bryantsev, V. S.; Shafer, J. C., Outer-Sphere Water Clusters Tune the Lanthanide Selectivity of Diglycolamides. ACS Cent. Sci. 2018, 4, 739-747.

34. Dufreche, J. F.; Zemb, T., Effect of Long-Range Interactions on Ion Equilibria in Liquid-Liquid Extraction. Chem. Phys. Lett. 2015, 622, 45-49.

35. Ferru, G.; Reinhart, B.; Bera, M. K.; Olvera de la Cruz, M.; Qiao, B.; Ellis, R. J., The Lanthanide Contraction beyond Coordination Chemistry. Chem . Eur. J. 2016, 22, 6899-6904.

36. Qiao, B. F.; Ferru, G.; de la Cruz, M. O.; Ellis, R. J., Molecular Origins of Mesoscale Ordering in a Metalloamphiphile Phase. ACS Cent. Sci. 2015, 1, 493-503.

37. Servis, M. J.; Wu, D. T.; Shafer, J. C.; Clark, A. E., Square Supramolecular Assemblies of Uranyl Complexes in Organic Solvents. Chem. Commun. 2018, 54, 10064-10067.

38. Sutton, J. E.; Roy, S.; Chowdhury, A. U.; Wu, L.; Wanhala, A. K.; De Silva, N.; Jansone-Popova, S.; Hay, B. P.; Cheshire, M. C.; Windus, T. L.; Stack, A. G.; Navrotsky, A.; Moyer, B. A.; Doughty, B.; Bryantsev, V. S., Molecular Recognition at Mineral Interfaces: Implications for the Beneficiation of Rare Earth Ores. ACS Appl. Mater. Interfaces 2020, 12, 16327-16341.

39. Nelson, J. J. M.; Schelter, E. J., Sustainable Inorganic Chemistry: Metal Separations for Recycling. Inorg. Chem. 2019, 58, 979-990.

40. Wilson, A. M.; Bailey, P. J.; Tasker, P. A.; Turkington, J. R.; Grant, R. A.; Love, J. B., Solvent Extraction: The Coordination Chemistry Behind Extractive Metallurgy. Chem. Soc. Rev. 2014, 43, 123134.

41. Wu, H.; Jones, L. O.; Wang, Y.; Shen, D.; Liu, Z.; Zhang, L.; Cai, K.; Jiao, Y.; Stern, C. L.; Schatz, G. C.; Stoddart, J. F., High-Efficiency Gold Recovery Using Cucurbit[6]uril. ACS Appl. Mater. Interfaces 2020, 12, 38768-38777.

42. Bernardis, F. L.; Grant, R. A.; Sherrington, D. C., A Review of Methods of Separation of the Platinum-Group Metals through Their Chloro-complexes. Reactive \& Functional Polymers 2005, 65, 205217.

43. Jha, M. K.; Gupta, D.; Lee, J. C.; Kumar, V.; Jeong, J., Solvent Extraction of Platinum Using Amine based Extractants in Different Solutions: A review. Hydrometallurgy 2014, 142, 60-69.

44. Peng, C. Y.; Tsai, T. H., Solvent Extraction of Palladium(II) from Acidic Chloride Solutions Using Tri-octyl/decyl Ammonium Chloride (Aliquat 336). Desalination Water Treat. 2014, 52, 1101-1108.

45. Iqbal, M.; Waheed, K.; Rahat, S. B.; Mehmood, T.; Lee, M. S., An Overview of Molecular Extractants in Room Temperature lonic Liquids and Task Specific lonic Liquids for the Partitioning of Actinides/Lanthanides. J. Radioanal. Nucl. Ch. 2020, 325, 1-31.

46. Binnemans, K., Lanthanides and Actinides in Ionic Liquids. Chem. Rev. 2007, 107, 2592-2614.

47. Lee, J. Y.; Kumar, J. R.; Kim, J. S.; Kim, D. J.; Yoon, H. S., Extraction and Separation of Pt(IV)/Rh(III) from Acidic Chloride Solutions Using Aliquat 336. J. Ind. Eng. Chem. 2009, 15, 359-364.

48. Lee, J. Y.; Raju, B.; Kumar, B. N.; Kumar, J. R.; Park, H. K.; Reddy, B. R., Solvent Extraction Separation and Recovery of Palladium and Platinum from Chloride Leach Liquors of Spent Automobile Catalyst. Sep. Purif. Technol. 2010, 73, 213-218. 
49. Marinho, R. S.; Afonso, J. C.; da Cunha, J., Recovery of Platinum from Spent Catalysts by LiquidLiquid Extraction in Chloride Medium. J. Hazard. Mater. 2010, 179, 488-494.

50. Knight, A. W.; Chiarizia, R.; Soderholm, L., Extraction Selectivity of a Quaternary Alkylammonium Salt for Trivalent Actinides over Trivalent Lanthanides: Does Extractant Aggregation Play a Role? Solvent Extr. Ion Exc. 2017, 35, 266-279.

51. Onghena, B.; Papagni, E.; Souza, E. R.; Banerjee, D.; Binnemans, K.; Vander Hoogerstraete, T., Speciation of Lanthanide lons in the Organic Phase after Extraction from Nitrate Media by Basic Extractants. RSC Adv. 2018, 8, 32044-32054.

52. Narita, H.; Nicolson, R. M.; Motokawa, R.; Ito, F.; Morisaku, K.; Goto, M.; Tanaka, M.; Heller, W. T.; Shiwaku, H.; Yaita, T.; Gordon, R. J.; Love, J. B.; Tasker, P. A.; Schofield, E. R.; Antonio, M. R.; Morrison, C. A., Proton Chelating Ligands Drive Improved Chemical Separations for Rhodium. Inorg. Chem. 2019, 58, 8720-8734.

53. Doidge, E. D.; Carson, I.; Love, J. B.; Morrison, C. A.; Tasker, P. A., The Influence of the Hofmeister Bias and the Stability and Speciation of Chloridolanthanates on Their Extraction from Chloride Media. Solvent Extr. Ion Exc. 2016, 34, 579-593.

54. Lommelen, R.; Vander Hoogerstraete, T.; Onghena, B.; Billard, I.; Binnemans, K., Model for Metal Extraction from Chloride Media with Basic Extractants: A Coordination Chemistry Approach. Inorg. Chem. 2019, 58, 12289-12301.

55. Mishra, P. K.; Chakravortty, V.; Dash, K. C.; Das, N. R.; Bhattacharyya, S. N., Extraction and Separation of $\mathrm{Zr}, \mathrm{Nb}$, and $\mathrm{Hf}$ by Aliquat-336 and its Mixtures with TOPO from Acidic Thiocyanate Media. J. Radioanal. Nucl. Chem. 1992, 162, 289-298.

56. Cerna, M.; Bizek, V.; Stastova, J.; Rod, V., Extraction of Nitric Acid with Quaternary Ammonium Bases. Chem. Eng. Sci. 1993, 48, 99-103.

57. Chiarizia, R.; Gatrone, R. C.; Horwitz, E. P., Am(III) and Eu(III) Extraction by Aliquat-336 and Benzyl Substituted Quaternary Ammonium-salts from Nitrate and Thiocyanate Solutions. Solvent Extr. Ion Exc. 1995, 13, 615-645.

58. Bell, K. J.; Westra, A. N.; Warr, R. J.; Chartres, J.; Ellis, R.; Tong, C. C.; Blake, A. J.; Tasker, P. A.; Schroder, M., Outer-Sphere Coordination Chemistry: Selective Extraction and Transport of the PtCl6 (2-) anion. Angew. Chem. Int. Ed. 2008, 47, 1745-1748.

59. Nayak, S.; Lovering, K.; Uysal, A., Ion-Specific Clustering of Metal-Amphiphile Complexes in Rare Earth Separations. Nanoscale 2020, 12, 20202-20210.

60. Bera, M. K.; Bu, W.; Uysal, A., Liquid Surface X-Ray Scattering. In Physical Chemistry of Gas-Liquid Interfaces, Faust, J. A.; House, J. E., Eds. Elsevier: 2018; pp 167-194.

61. Clark, A. E.; Yang, P.; Shafer, J. C., Coordination of Actinides and the Chemistry Behind Solvent Extraction. In Experimental and Theoretical Approaches to Actinide Chemistry, 2018; pp 237-282.

62. Paiva, A. P., Recycling of Palladium from Spent Catalysts Using Solvent Extraction-Some Critical Points. Metals 2017, 7, 505.

63. Ellis, R. J., Acid-Switched Eu(III) Coordination inside Reverse Aggregates: Insights into a Synergistic Liquid-Liquid Extraction System. Inorganica Chim. Acta 2017, 460, 159-164.

64. Sun, P.; Huang, K.; Liu, H. Z., Specific Salt Effect on the Interaction between Rare Earth lons and Trioctylphosphine Oxide Molecules at the Organic-Aqueous Two-Phase Interface: Experiments and Molecular Dynamics Simulations. Langmuir 2018, 34, 11374-11383.

65. Nayak, S.; Lovering, K.; Bu, W.; Uysal, A., Anions Enhance Rare Earth Adsorption at Negatively Charged Surfaces. J. Phys. Chem. Lett. 2020, 4436-4442.

66. Miller, M.; Liang, Y. H.; Li, H. H.; Chu, M. Q.; Yoo, S. J.; Bu, W.; de la Cruz, M. O.; Dutta, P., Electrostatic Origin of Element Selectivity during Rare Earth Adsorption. Phys. Rev. Lett. 2019, 122, 6. 
67. Clark, A. E., Amphiphile-Based Complex Fluids: The Self-Assembly Ensemble as Protagonist. ACS Cent. Sci. 2019, 5, 10-12.

68. Li, X. D.; Charaya, H.; Bernard, G. M.; Elliott, J. A. W.; Michaelis, V. K.; Lee, B.; Chung, H. J., LowTemperature Ionic Conductivity Enhanced by Disrupted Ice Formation in Polyampholyte Hydrogels. Macromolecules 2018, 51, 2723-2731.

69. Ruckenstein, E.; Nagarajan, R., Aggregation of amphiphiles in nonaqueous media. J. Phys. Chem. 1980, 84, 1349-1358.

70. Harton, S. E.; Pingali, S. V.; Nunnery, G. A.; Baker, D. A.; Walker, S. H.; Muddiman, D. C.; Koga, T.; Rials, T. G.; Urban, V. S.; Langan, P., Evidence for Complex Molecular Architectures for Solvent-Extracted Lignins. ACS Macro Lett. 2012, 1, 568-573.

71. Naidoo, K. J.; Klatt, G.; Koch, K. R.; Robinson, D. J., Geometric Hydration Shells for Anionic Platinum Group Metal Chloro Complexes. Inorg. Chem. 2002, 41, 1845-1849.

72. Uehara, T. M.; Marangoni, V. S.; Pasquale, N.; Miranda, P. B.; Lee, K. B.; Zucolotto, V., A Detailed Investigation on the Interactions between Magnetic Nanoparticles and Cell Membrane Models. ACS Appl. Mater. Interfaces 2013, 5, 13063-13068.

73. Bernhard, C.; Bauer, K. N.; Bonn, M.; Wurm, F. R.; Gonella, G., Interfacial Conformation of Hydrophilic Polyphosphoesters Affects Blood Protein Adsorption. ACS Appl. Mater. Interfaces 2019, 11, 1624-1629.

74. Lovering, K.; Nayak, S.; Bu, W.; Uysal, A., The Role of Specific lon Effects in Ion Transport: The Case of Nitrate and Thiocyanate. J. Phys. Chem. C 2019, 124, 573-581.

75. Leontidis, E., Investigations of the Hofmeister Series and other Specific lon Effects Using Lipid Model Systems. Adv. Colloid Interface Sci. 2017, 243, 8-22.

76. Eisenthal, K. B., Liquid Interfaces Probed by Second-Harmonic and Sum-Frequency Spectroscopy. Chem. Rev. 1996, 96, 1343-1360.

77. Chowdhury, A. U.; Lin, L.; Doughty, B., Hydrogen-Bond-Driven Chemical Separations: Elucidating the Interfacial Steps of Self-Assembly in Solvent Extraction. ACS Appl. Mater. Interfaces 2020, 12, 3211932130.

78. Johnson, C. M.; Baldelli, S., Vibrational Sum Frequency Spectroscopy Studies of the Influence of Solutes and Phospholipids at Vapor/Water Interfaces Relevant to Biological and Environmental Systems. Chem. Rev. 2014, 114, 8416-8446.

79. Bonn, M.; Nagata, Y.; Backus, E. H. G., Molecular Structure and Dynamics of Water at the WaterAir Interface Studied with Surface-Specific Vibrational Spectroscopy. Angew. Chem. Int. Ed. 2015, 54, 5560-5576.

80. Baumler, S. M.; Allen, H. C., Vibrational Spectroscopy of Gas-Liquid Interfaces. In Physical Chemistry of Gas-Liquid Interfaces, Faust, J. A.; House, J. E., Eds. Elsevier: 2018; pp 105-133.

81. Bourgeois, D.; El Maangar, A.; Dourdain, S., Importance of Weak Interactions in the Formulation of Organic Phases for Efficient Liquid/Liquid Extraction of Metals. Curr. Opin. Colloid Interface Sci. 2020, 46, 36-51.

82. Khopkar, P. K.; Mathur, J. N., Thiocyanate Complexing of Some Trivalent Actinides and Lanthanides. J. Inorg. Nucl. Chem. 1974, 36, 3819-3825.

83. Horwitz, E. P.; Muscatello, A. C.; Kalina, D. G.; Kaplan, L., The Extraction of Selected Transplutonium(III) and Lanthanide(III) lons by Dihexyl-N, N-diethylcarbamoylmethylphosphonate from Aqueous Nitrate Media. Sep. Sci. Technol. 1981, 16, 417-437.

84. Li, Z.; Binnemans, K., Hydration Counteracts the Separation of Lanthanides by Solvent Extraction. AlChE Journal 2020, 66, e16545.

85. Ramanathan, G. V., Counterion Condensation in Micellar and Colloidal Solutions. J. Chem. Phys. $1988,88,3887-3892$. 
86. Faeder, J.; Albert, M. V.; Ladanyi, B. M., Molecular Dynamics Simulations of the Interior of Aqueous Reverse Micelles: A Comparison Between Sodium and Potassium Counterions. Langmuir 2003, 19, 2514-2520.

87. Aswal, V. K.; Goyal, P. S., Role of Different Counterions and Size of Micelle in Concentration Dependence Micellar Structure of lonic Surfactants. Chem. Phys. Lett. 2003, 368, 59-65.

88. Aswal, V. K.; Kohlbrecher, J.; Goyal, P. S.; Amenitsch, H.; Bernstorff, S., Counterion Condensation on Charged Micelles in an Aqueous Electrolyte Solution as Studied with Combined Small-Angle Neutron Scattering and Small-Angle X-ray Scattering. J. Phys. Condens. Matter 2006, 18, 11399-11410.

89. Geng, Y.; Romsted, L. S.; Menger, F., Specific Ion Pairing and Interfacial Hydration as Controlling Factors in Gemini Micelle Morphology. Chemical Trapping Studies. J. Am. Chem. Soc. 2006, 128, 492501.

90. Pott, T.; Meleard, P., New Insight into the Nanostructure of Ionic Liquids: a Small Angle X-ray Scattering (SAXS) Study on Liquid Tri-alkyl-methyl-ammonium bis(trifluoromethanesulfonyl)amides and their Mixtures. Phys. Chem. Chem. Phys. 2009, 11, 5469-5475.

91. Ma, C. Y.; Laaksonen, A.; Liu, C.; Lu, X. H.; Ji, X. Y., The Peculiar Effect of Water on lonic Liquids and Deep Eutectic Solvents. Chem. Soc. Rev. 2018, 47, 8685-8720.

92. Verma, A.; Stoppelman, J. P.; McDaniel, J. G., Tuning Water Networks via lonic Liquid/Water Mixtures. Int. J. Mol. Sci. 2020, 21, 403.

93. Wang, Y. L.; Li, B.; Sarman, S.; Mocci, F.; Lu, Z. Y.; Yuan, J. Y.; Laaksonen, A.; Fayer, M. D., Microstructural and Dynamical Heterogeneities in lonic Liquids. Chem. Rev. 2020, 120, 5798-5877.

94. Lambert, A. G.; Davies, P. B.; Neivandt, D. J., Implementing the Theory of Sum Frequency Generation Vibrational Spectroscopy: A Tutorial Review. Applied Spectroscopy Reviews 2005, 40, 103145.

95. Abraham, M. J.; Murtola, T.; Schulz, R.; Páll, S.; Smith, J. C.; Hess, B.; Lindahl, E., GROMACS: High Performance Molecular Simulations through Multi-level Parallelism from Laptops to Supercomputers. SoftwareX 2015, 1-2, 19-25.

96. Best, R. B.; Zhu, X.; Shim, J.; Lopes, P. E. M.; Mittal, J.; Feig, M.; MacKerell, A. D., Optimization of the Additive CHARMM All-Atom Protein Force Field Targeting Improved Sampling of the Backbone phi, psi and Side-Chain chi(1) and chi(2) Dihedral Angles. J. Chem. Theory Comput. 2012, 8, 3257-3273.

97. Lienke, A.; Klatt, G.; Robinson, D. J.; Koch, K. R.; Naidoo, K. J., Modeling Platinum Group Metal Complexes in Aqueous Solution. Inorg. Chem. 2001, 40, 2352-2357.

98. Jorgensen, W. L.; Chandrasekhar, J.; Madura, J. D.; Impey, R. W.; Klein, M. L., Comparison of Simple Potential Functions for Simulating Liquid Water. J. Chem. Phys. 1983, 79, 926-935.

99. Parrinello, M.; Rahman, A., Polymorphic Transitions in Single-Crystals - A New MolecularDynamics Method. J. App. Phys. 1981, 52, 7182-7190.

100. Hoover, W. G., Canonical Dynamics - Equilibrium Phase-Space Distributions. Phys. Rev. A 1985, 31, 1695-1697.

101. Darden, T.; York, D.; Pedersen, L., Particle Mesh-Ewald - An N.log(N) Method for Ewald Sums in Large Systems. J. Chem. Phys. 1993, 98, 10089-10092. 


\section{TABLE OF CONTENTS FIGURE}

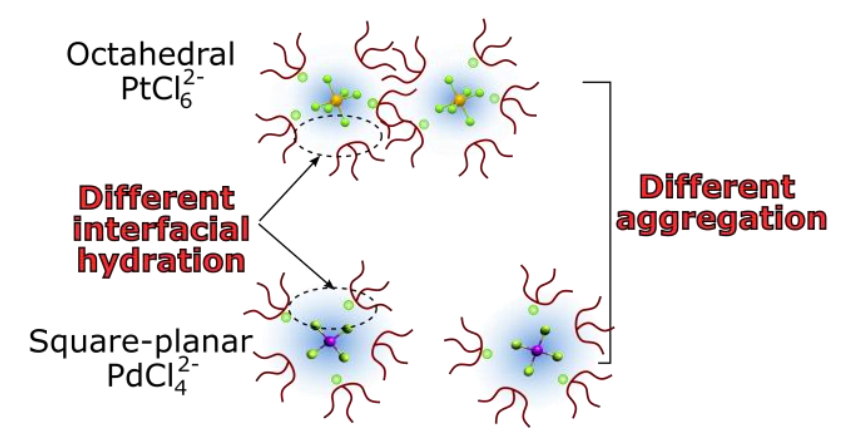

